\title{
Kompetenzen und Wege der Kompetenzentwicklung in der Industrie 4.0
}

\author{
Verena Simone Lore Blumberg' 1 - Simone Kauffeld ${ }^{1}$
}

Angenommen: 22. April 2021 / Online publiziert: 12. Mai 2021

(c) Der/die Autor(en) 2021

\section{Zusammenfassung}

Die fortschreitende Digitalisierung verändert die Arbeitswelt auch in der industriellen Fertigung nachhaltig. Digitale Werkerassistenzsysteme wie Datenbrillen und Smartwatches unterstützen Mitarbeitende mit kontextsensitiven Informationen. Die Einführung dieser Technologien und die Veränderung von Arbeitsabläufen stellen neue Kompetenzanforderungen an Beschäftigte, ermöglichen aber auch veränderte Wege des Lernens. Ziel dieses Beitrags der Zeitschrift Gruppe. Interaktion. Organisation. (GIO) ist es, zukünftig benötigte Kompetenzen für Beschäftigte in der Produktion sowie mögliche Wege der Kompetenzentwicklung zu identifizieren. Dazu wurden 76 halbstrukturierte Interviews mit Teilnehmenden aus den Expertenclustern Wissenschaft, Politik und betriebliche Praxis durchgeführt und inhaltsanalytisch ausgewertet. Als Ergebnisse der Studie ist ein Kompetenzmodell für Beschäftigte in der Produktion entstanden, das auf der etablierten Unterscheidung in die Kompetenzfacetten Fach-, Methoden-, Sozial- und Selbstkompetenz aufbaut. Zusätzlich wurde die digitale Kompetenz als Querschnittskompetenz ergänzt. Besonders relevant wird über alle Expertengruppen hinweg die IT-Anwendungskompetenz eingeschätzt. Bei den identifizierten Wegen der Kompetenzentwicklung werden zunehmend selbstinitiierte Lernformate bedeutsam, die die Möglichkeiten der Digitalisierung nutzen. Die vorliegenden Ergebnisse können als Ausgangspunkt für die Entwicklung betrieblicher Kompetenzmodelle und -entwicklungsprogramme genutzt werden.

Schlüsselwörter Digitalisierung $\cdot$ Industrie $4.0 \cdot$ Kompetenzen $\cdot$ Qualifizierung $\cdot$ Produktion

\section{Competencies and competence development in industry 4.0}

\section{Abstract}

The advance in digitization is also changing the way of working in the field of industrial manufacturing. Smart wearables and mobile smart devices e.g. smart glasses and smartwatches, support employees with context-sensitive information. The introduction of these technologies and the changes in work processes lead to new competence requirements for employees and new paths for competence development. This article in the journal Gruppe. Interaktion. Organisation. (GIO) aims to identify future competence requirements and ways of competence development for industrial workers. Therefore, 76 semi-structured interviews with participants from five expert groups were conducted. As a result, our study presents a competence model which builds on the well-established structure of professional, methodological, social and personal competences. Digital competences were added as cross-sectional competence. General IT application skills were identified as a core competence for employees in the industry 4.0. For competence development digital and self-initiated ways of learning are getting more important. The results of this study can be used to develop an operational competence management.

Keywords Digitization $\cdot$ Smart wearables $\cdot$ Industry $4.0 \cdot$ Competencies $\cdot$ Qualifications $\cdot$ Manufacturing

Verena Simone Lore Blumberg, M.Sc.

v.blumberg@tu-braunschweig.de

Prof. Dr. Simone Kauffeld

s.kauffeld@tu-braunschweig.de
1 Lehrstuhl für Arbeits-, Organisations- und Sozialpsychologie, Technische Universität Braunschweig, Spielmannstr. 19, 38106 Braunschweig, Deutschland 


\section{Einleitung}

Neue Technologien durchdringen die Arbeitswelt in einem immer erheblicheren Maße und werden zu allgegenwärtigen Begleitern der Beschäftigten (Cascio und Montealegre 2016). Dies betrifft nicht mehr nur die Wissensberufe oder hochautomatisierte Produktionsabläufe, sondern trifft in einem erheblichen Maße auch Berufsbereiche, die bisher noch nicht direkt von der Anwendung der Informations- und Kommunikationstechnologien betroffen waren, wie beispielsweise die industrielle Fertigung. In der industriellen Fertigung wird die neue Durchdringung und Allgegenwärtigkeit von Digitalisierung mit dem Schlagwort Industrie 4.0 beschrieben (Lasi et al. 2014; Promotorengruppe Kommunikation der Forschungsunion Wirtschaft Wissenschaft 2013). Ein zentrales Element dieser zunehmenden Vernetzung sind digitale Werkerassistenzsysteme (DWAS), beispielsweise Datenbrillen, Tablets oder Smartwatches (Evers et al. 2019). DWAS werden definiert als mobile digitale Endgeräte, die körpernah getragen werden und Mitarbeitende bei der Ausführung der Tätigkeit durch Informationsdarbietung unterstützen (Niehaus 2017). Mit DWAS können Mitarbeitende in die neue, vernetzte Arbeitsumgebung eingebunden werden. Die Anwendungsmöglichkeiten für DWAS in der industriellen Fertigung sind vielfältig. Beispielsweise können DWAS zum Arbeitsschutz, zur temporären oder dauerhaften Unterstützung der Arbeitstätigkeit, zur Kommunikation, zur Qualitätssicherung oder zur Qualifizierung eingesetzt werden (Blumberg und Kauffeld 2020). Damit die Potenziale der neuen Technologien ausgeschöpft werden können, kommt den Produktionsbeschäftigten als Anwendenden dieser Technologien eine hohe Bedeutung zu (Güth et al. 2018).

Die zunehmende Digitalisierung ist dabei sowohl Auslöser für die Veränderung benötigter Kompetenzen als auch anwendbares Mittel, um die Kompetenzentwicklung zu gestalten (Bornewasser et al. 2018). Neben der zunehmenden Digitalisierung machen auch sich verändernde Arbeitsbedingungen neue bzw. andere Kompetenzen bei den Beschäftigten erforderlich (Acatech 2016; Botthof 2015). Beschäftigte werden stärker als bisher damit konfrontiert, sich kontinuierlich weiterzubilden, um sowohl das fachliche als auch das methodische Wissen aktuell zu halten (Leimeister und David 2019a; Metternich et al. 2018). Dies wird ohne den zeitgleichen Aufbau von Sozial- und Selbstkompetenzen nicht leistbar sein. Um den neuen Anforderungen gerecht zu werden und die notwendigen Kompetenzen frühzeitig aufzubauen, ist es erforderlich, die notwendigen Kompetenzanforderungen an Beschäftigte in der Produktion zu erfassen und Wege der Kompetenzentwicklung aufzuzeigen.

\section{Kompetenzen und ihre systematische Entwicklung}

Obwohl die Veränderung von Kompetenzen seit längerer Zeit ein relevantes Thema in der Gesellschaft und unterschiedlichen wissenschaftlichen Disziplinen ist, gibt es nach wie vor keinen Konsens über eine einheitliche Definition des Konstruktes (Brown 1993; Erpenbeck et al. 2017; Le Deist und Winterton 2005; Kauffeld und Paulsen 2018; Moore et al. 2002; Mulder 2017). International kann zwischen einer eher spezifisch verhaltensorientierten und einer ganzheitlich integrativen Betrachtung von Kompetenzen unterschieden werden (Bornewasser et al. 2018; Brockmann et al. 2008; Misbah et al. 2019). Ein spezifisch verhaltensorientiertes Verständnis von Kompetenz stellt vor allem die erfolgreiche Leistung in einer konkreten Arbeitssituation in den Mittelpunkt der Betrachtung. Dies ist auch in der nachfolgenden Definition des Kompetenzbegriffs durch die International Organization for Standardization (ISO) erkennbar. Hier heißt es: „, [competences are] the ability to apply knowledge and skills to achieve intended results" (ISO 2015). Zentral ist also die praktische Fähigkeit, Wissen und Fähigkeiten anzuwenden (,ability to apply“, ISO 2015), um bestimmte Ziele $\mathrm{zu}$ erreichen. Bei der eher ganzheitlich und integrativen Betrachtung des Kompetenzbegriffs stehen vor allem die kognitiven Ressourcen im Fokus, die zielorientiertes Handeln im Arbeitskontext ermöglichen (Bornewasser et al. 2018). Dies zeigt auch die nachfolgende Definition: Unter Kompetenzen werden sowohl das Wissen als auch alle Fähigkeiten und Fertigkeiten einer Person verstanden, die diese bei der Bewältigung bekannter und neuartiger Situationen handlungs- und reaktionsfähig machen (Kauffeld 2006). Beide Definitionen eint, dass nicht nur das Wissen (vgl. Fachkompetenz), sondern ebenso weitere Fähigkeiten und Fertigkeiten (vgl. Methoden-, Sozial- und Selbstkompetenz) für das erfolgreiche Handeln als relevant angenommen werden. Die besondere Bedeutung und Relevanz von Kompetenzen im betrieblichen Kontext ergibt sich daraus, dass Kompetenzen zu einer höheren Arbeitsqualität führen, systematisch und aufwendig entwickelt werden müssen und nur schwer imitiert werden können, wenn sie einmal aufgebaut sind. Kompetenzen stellen somit einen besonderen Wettbewerbsvorteil dar (Kauffeld 2006; Kauffeld und Paulsen 2018).

Wie Kompetenzanforderungen, Kompetenzmodelle und Kompetenzentwicklung zusammenspielen können, ist in Abb. 1 dargestellt. Um Kompetenzanforderungen an Beschäftigte zu erfassen, kommt dem strategischen Kompetenzmanagement eine besondere Bedeutung zu. Dabei werden Kompetenzentwicklungsbedarfe aus Trends im Unternehmensumfeld, den Zielen des Unternehmens und der Anpassung der Unternehmensstrukturen und Geschäftspro- 
Abb. 1 Vereinfachte Ablaufdarstellung des betrieblichen Kompetenzmanagements

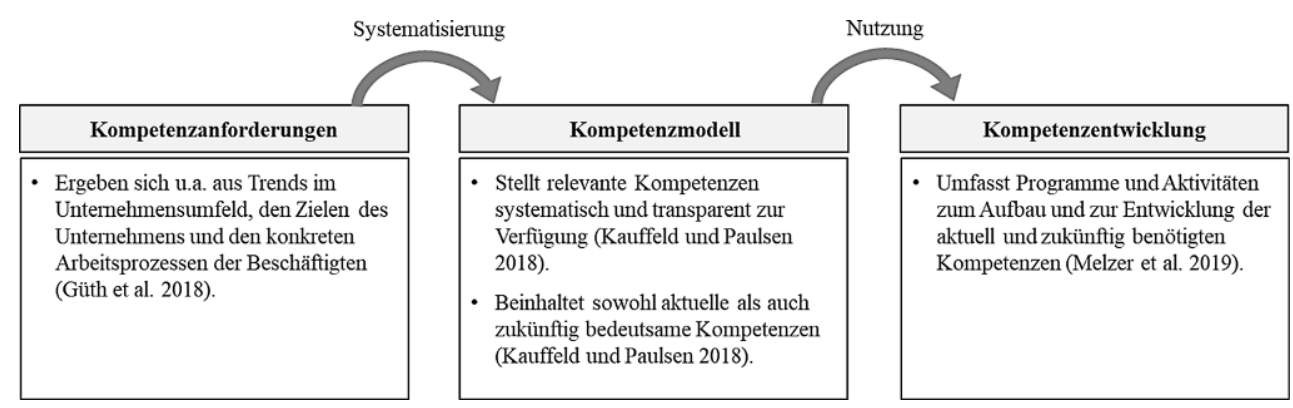

zesse abgeleitet. Zusätzlich werden konkrete Tätigkeitsanforderungen berücksichtigt, die aus den Verantwortlichkeiten der Beschäftigten sowie erwarteter Veränderungen der Arbeitsabläufe und Aufgabeninhalte abgeleitet werden (Güth et al. 2018). Dabei ist im Unterschied zur klassischen Arbeits- und Anforderungsanalyse nicht der Inhalt der einzelnen Aufgaben relevant (Das „Was“), sondern wie diese zielführend und erfolgreich bewältigt werden können (Das „Wie“) (Kauffeld und Paulsen 2018; Shippmann et al. 2000). Die zukünftig stärkere Nutzung von DWAS in der industriellen Produktion ist ein sichtbares Zeichen der zunehmenden Digitalisierung und ein aktueller Trend, der zu neuen und veränderten Kompetenzbedarfen bei Produktionsbeschäftigten führen kann.

Zur Systematisierung der abgeleiteten Kompetenzanforderungen werden Kompetenzmodelle genutzt (Kauffeld und Paulsen 2018). In Kompetenzmodellen werden sowohl aktuell bedeutsame als auch zukünftig relevante Kompetenzen berücksichtigt (Kauffeld und Paulsen 2018). Damit werden Anforderungen an Beschäftigte einer bestimmten Zielgruppe sichtbar und transparent gemacht. Eine Ordnungsstruktur für die identifizierten Kompetenzen bietet beispielsweise die etablierte Unterteilung in die vier Kompetenzfacetten Fach-, Methoden-, Sozial- und Selbstkompetenz (vgl. Kauffeld 2006; Kauffeld und Paulsen 2018; Sonntag und Schaper 1999). Kompetenzmodelle stellen oft ein Bindeglied zwischen verschiedenen Human-ResourceProzessen dar und tragen zu einer effektiven Personalarbeit bei. Ausgehend von Kompetenzmodellen werden weiterführende Maßnahmen des betrieblichen Kompetenzmanagements angestoßen, beispielsweise Maßnahmen des Personalmarketings, der Personalauswahl und der Kompetenzentwicklung (Kauffeld und Grote 2019; Kauffeld und Paulsen 2018).

In der industriellen Produktion werden für einfache Tätigkeiten (z. B. Montagetätigkeiten, Logistik) an vielen Stellen an- und ungelernte Beschäftigte eingesetzt. Viele dieser Tätigkeiten werden manuell durchgeführt und sind daher sehr personalintensiv (Hinrichsen et al. 2016). In unserem Beitrag fokussieren wir auf Beschäftigte in den Gewerken Montage und Logistik, die einfache Tätigkeiten ausführen. Einfache Tätigkeiten sind meist durch einen hohen Standar- disierungsgrad gekennzeichnet (Bouville und Alis 2014), dennoch sind für die Ausführung der Tätigkeiten bestimmte Kompetenzen und Qualifikationen unabdingbar (Bellmann et al. 2015; Schöpper-Grabe und Vahlhaus 2019). Bei Untersuchungen zum strategischen Kompetenzmanagement konnte gezeigt werden, dass in klein- und mittelständischen Unternehmen nur bei knapp 50\% der befragten Unternehmen Aktivitäten zum strategischen Kompetenzmanagement für an- und ungelernte Beschäftigte stattfinden. Diese Aktivitäten umfassen die Erstellung von Anforderungsprofilen, die systematische Erfassung bestehender Kompetenzen und die Verfügbarkeit von Programmen zur Kompetenzentwicklung. Für die Zielgruppe der Fachkräfte werden hingegen in knapp 90\% der Unternehmen Aktivitäten zum strategischen Kompetenzmanagement unternommen (Güth et al. 2018). Dies zeigt, dass es für die Zielgruppe der an- und ungelernten Beschäftigten einen erheblichen Bedarf und ein hohes Potenzial für die Verbesserung des strategischen Kompetenzmanagements gibt.

Wie in Abb. 1 dargestellt, schließt an die Identifikation der relevanten Kompetenzen die Kompetenzentwicklung mit den dazugehörigen Prozessen an, die den systematischen Aufbau der benötigten Kompetenzen für die jeweilige Arbeitstätigkeit fördert (Melzer et al. 2019). Kompetenzentwicklungsmaßnahmen lassen sich anhand des Organisationsgrades (Ist die Aktivität als Lernaktivität geplant?) und der Lernintention (Ist Lernen intendiert?) in formale, non-formale und informelle Kompetenzentwicklung unterscheiden (OECD 2007; Kauffeld und Paulsen 2018). Diese Unterscheidung ist in Abb. 2 dargestellt.

\subsection{Kompetenzen in der digitalisierten Arbeitswelt}

In einer digitalisierten Arbeitswelt verändern sich die Anforderungen, die an Beschäftigte gestellt werden. So verweisen beispielsweise Windelband und Dworschak (2015) darauf, dass die Industrie 4.0 Fachkräfte braucht, die in der Lage sind, in hochkomplexen, wandlungsfähigen und flexiblen Produktionssystemen Entscheidungen zu treffen und zu steuern, die Systeme instand zu halten und als Experten zu agieren. Dies zieht die Frage nach sich, welche Kompetenzen dafür erforderlich sind. 


\begin{tabular}{|c|c|c|c|}
\hline & Formale Kompetenzentwicklung & Non-formale Kompetenzentwicklung & Informelle Kompetenzentwicklung \\
\hline Organisationsgrad & - Stets organisiert und strukturiert & - Eher organisiert & - Nicht organisiert \\
\hline Lernintention & $\begin{array}{l}\text { - Lernen ist beabsichtigt } \\
\text { - Lernziele sind expliziert und bekannt }\end{array}$ & $\begin{array}{l}\text { - Lernen ist teilweise intendiert } \\
\text { - Lernziele werden teilweise expliziert }\end{array}$ & $\begin{array}{l}\text { - Lernen ist nicht intendiert oder } \\
\text { zielgerichtet, sondern eher beiläufig } \\
\text { - Lernziele sind nicht expliziert }\end{array}$ \\
\hline Lernort & $\begin{array}{l}\text { Meist vom Arbeitsort getrennt, } \\
\text { z. B. Seminare, Kurse }\end{array}$ & $\begin{array}{l}\text { Eher im Prozess der Arbeit, } \\
\text { z. B. Anlernen, Unterweisungen }\end{array}$ & $\begin{array}{l}\text { - Unterschiedlich } \\
\text { z. B. soziale Situationen (Lernen von } \\
\text { anderen Personen) oder Reflexion } \\
\text { (Lernen von sich selbst) }\end{array}$ \\
\hline
\end{tabular}

Abb. 2 Wege der Kompetenzentwicklung

Nachfolgend werden bestehende Befunde zur Veränderung der Kompetenzanforderungen in der digitalisierten Arbeitswelt entlang der vier Facetten Fach-, Methoden-, Sozial- und Selbstkompetenz vorgestellt und Hypothesen abgeleitet. Die Anforderungen an digitale Kompetenzen werden anschließend separat betrachtet. Wir gehen davon aus, dass die etablierte Unterscheidung in Fach-, Methoden-, Sozial- und Selbstkompetenz auch für die Systematisierung der Kompetenzanforderungen an Produktionsbeschäftigte in der Industrie 4.0 ein geeignetes Rahmenmodell bietet und die identifizierten Kompetenzanforderungen diesen Facetten zugeordnet werden können (Hypothese 1).

\subsubsection{Fachkompetenz in der digitalisierten Arbeitswelt}

Der Fachkompetenz werden aufgaben- und arbeitsplatzspezifische Fertigkeiten, Fähigkeiten und Kenntnisse zugeordnet (vgl. Kauffeld 2006; Kauffeld und Paulsen 2018). Diese sind meist disziplinspezifisch und somit auf eine bestimmte berufliche Tätigkeit ausgerichtet. Veränderte Arbeitsprozesse und -abläufe haben daher häufig die Anpassung der Fachkompetenz zur Folge. Ebenso können daraus auch Veränderungen der überfachlichen Kompetenzen resultieren.

In der industriellen Produktionsarbeit werden Materialien und Güter verarbeitet, montiert, erstellt und das Ergebnis kontrolliert (Elkmann et al. 2015). Diese grundsätzliche Anforderung wird auch bei der Einführung von DWAS, die den Beschäftigten mit der Bereitstellung von Informationen assistieren, als elementarer Bestandteil der Arbeitsaufgabe erhalten bleiben (Baethge-Kinsky 2020). Erste Untersuchungen zeigen zwar, dass in Unternehmen mit einem hohen Digitalisierungsgrad handwerkliche Fähigkeiten und technisches Fachwissen insgesamt als weniger bedeutsam eingeschätzt werden (Hammermann und Stettes 2016), dennoch wird den Kompetenzen für bestimmte Mitarbeitergruppen, wie Produktionsmitarbeitenden, eine weiterhin hohe Bedeutung zugesprochen (Baethge-Kinsky 2020; Hammermann und Stettes 2016).
Weiterhin steigt die Anforderung an Beschäftigte, die eigenen Prozesse und Ergebnisse in die digitalisierte und automatisierte Arbeitsumgebung einordnen zu können (Hammermann und Stettes 2016). Die Beschreibung dieser Anforderungen wird in der Literatur unter der Kompetenz Prozessdenken zusammengefasst. Prozessdenken beschreibt ein fachliches Verständnis für vor- und nachgelagerte Schritte im Arbeitsprozess. Dazu gehört auch, die Folgen des eigenen Handelns auf diese abschätzen zu können (Hillebrand 2018). Da bei dieser Anforderung das Fachwissen über den gesamten Arbeitsprozess (eigene Tätigkeit inkl. vor- und nachgelagerter Prozessschritte) und das fachliche Verständnis zum Ineinandergreifen der unterschiedlichen Arbeitsschritte im Fokus stehen, wird diese Kompetenz der Facette Fachkompetenz zugeordnet.

Aufbauend auf den dargestellten Untersuchungsergebnissen gehen wir davon aus, dass als relevante Kompetenzen in der Facette Fachkompetenz mindestens die Kompetenzen Fachwissen, handwerkliche Fähigkeiten und Prozessdenken benannt werden (Hypothese 2).

\subsubsection{Methodenkompetenz in der digitalisierten Arbeitswelt}

Die Methodenkompetenz enthält Fähigkeiten und Fertigkeiten, die situationsübergreifend eingesetzt werden können. Diese Kompetenzen dienen der Strukturierung von Sachverhalten (vgl. Kauffeld 2006; Kauffeld und Paulsen 2018).

In einer immer komplexer und digitaler werdenden Arbeitsumgebung gewinnt die Fähigkeit, komplexe Probleme zu lösen, immer mehr an Bedeutung (Baethge-Kinsky 2020; Levy 2010; Promotorengruppe Kommunikation der Forschungsunion Wirtschaft - Wissenschaft 2013). Problemlösefähigkeit beschreibt alle Aktivitäten der Suche nach einer Lösung für ein Problem und ist ein mehrdimensionales Konstrukt (Schoppek und Fischer 2015). Es umfasst ein Set unterschiedlicher Fähigkeiten und Fertigkeiten, die genutzt werden, um nicht-routine Situationen erfolgreich zu bewäl- 
tigen (Fischer und Neubert 2015). Ein Kernaspekt dabei ist die Problemanalyse, bei der das Problem erfasst und Aufgaben und Zwischenziele für die Lösung des Problems entwickelt werden (Hesse et al. 2015). Erste Befunde zeigen, dass in digitalisierten Arbeitsumgebungen stärker als bisher eine theoriebasierte Herangehensweise bei der Problemlösung im Sinne einer strukturierten Problemanalyse und Datenanalyse notwendig wird (Baethge-Kinsky 2020). Außerdem gewinnt die sog. kollaborative Problemlösung an Bedeutung (Hesse et al. 2015), da komplexe Probleme häufig nicht mehr von einer Person allein bewältigt werden können, sondern das Zusammenarbeiten unterschiedlicher Personen erforderlich macht (Zeller et al. 2010). An dieser Stelle wird deutlich, dass die Methodenkompetenz Problemanalyse nicht nur eine Verknüpfung zur digitalen Kompetenz im Sinne einer Datenanalysekompetenz, sondern auch zur Sozialkompetenz aufweist.

Basierend auf den vorgestellten Befunden gehen wir davon aus, dass in der Facette Methodenkompetenz mindestens Analysefähigkeit als relevante Kompetenz benannt wird (Hypothese 3).

\subsubsection{Sozialkompetenz in der digitalisierten Arbeitswelt}

Die Sozialkompetenz umfasst Fähigkeiten und Fertigkeiten für die erfolgreiche Gestaltung und Bewältigung sozialer Interaktions- und Kooperationssituationen (vgl. Kauffeld 2006; Kauffeld und Paulsen 2018). Elementare Dimensionen dabei sind beispielsweise die Kommunikations-, Kooperations- und Koordinationsfähigkeit (Schaper 2004).

In der Diskussion um veränderte Kompetenzanforderungen in der Industrie 4.0 wurde vor allem eine zunehmende Bedeutung der kommunikativen Kompetenz prognostiziert (Promotorengruppe Kommunikation der Forschungsunion Wirtschaft - Wissenschaft 2013). Wie im vorherigen Abschnitt beschrieben, sind aufgrund digital-verketteter Prozesse viele der auftretenden Probleme nur in Zusammenarbeit mit anderen Personen lösbar. Dadurch gewinnt die erfolgreiche Gestaltung dieser Interaktionssituationen an Bedeutung. In Unternehmensfallstudien konnte der steigende Bedarf für Kommunikation und gegenseitige Unterstützung in der digitalen Arbeitswelt belegt werden (Baethge-Kinsky 2020). Weiterhin weisen erste Untersuchungen darauf hin, dass Mitarbeitende aufgrund von flexiblen Arbeitsorganisationen und dezentralen Entscheidungsstrukturen zukünftig mehr Kooperationsbereitschaft und eine stärkere Коттиnikationskompetenz benötigen (Hammermann und Stettes 2016).

Aufbauend auf der vorgestellten Literatur gehen wir davon aus, dass als relevante Kompetenzen in der Facette Sozialkompetenz mindestens Kommunikationsfähigkeit und Kooperationsfähigkeit benannt werden (Hypothese 4).

\subsubsection{Selbstkompetenz in der digitalisierten Arbeitswelt}

Die Selbstkompetenz beschreibt Fähigkeiten und Fertigkeiten, die zur Selbstorganisation und -steuerung genutzt werden (vgl. Kauffeld 2006; Kauffeld und Paulsen 2018). Dies umfasst in besonderem Maße Fähigkeiten, um den eigenen Arbeitsplatz konstruktiv mitzugestalten, zu organisieren und Verantwortung zu übernehmen (Kauffeld 2006). Diese Detaillierung zeigt, dass sowohl Aspekte der Motivation als auch der Verantwortung in dieser Facette verortet werden können.

Die gerade benannten Inhaltspunkte der Selbstkompetenz finden sich in ähnlicher Form in der aktuellen Diskussion um veränderte Kompetenzanforderungen in der Industrie 4.0 wieder. Hier wurde vor allem eine zunehmende Bedeutung von selbstgesteuertem Handeln und Selbstorganisation prognostiziert (Promotorengruppe Kommunikation der Forschungsunion Wirtschaft - Wissenschaft 2013) sowie eine höhere Bereitschaft, die eigenen Arbeitstätigkeiten eigenständig und eigenverantwortlich zu organisieren (Hammermann und Stettes 2016). Diese Ausführungen sprechen für einen höheren Bedarf, eigeninitiativ Verantwortung zu übernehmen, Entscheidungen zu treffen und für die Konsequenzen des eigenen Handelns einzustehen.

Dass Beschäftigte in ihrem Berufsleben immer häufiger mit Veränderungen konfrontiert sind, ergibt sich nicht nur aus der zunehmenden und neuen Art der Digitalisierung, sondern liegt auch in bereits bekannten und weiter anhaltenden Trends wie veränderten Produktionstechniken, immer kürzeren Produktlebenszyklen und einer stärkeren Individualisierung der Produkte begründet (Abele et al. 2017; Dillerup et al. 2019). Damit Beschäftigte bei diesen Veränderungen Schritt halten und sich immer wieder auf sich verändernde Rahmenbedingungen einstellen können, benötigen sie ein hohes $\mathrm{Ma} \beta$ an Veränderungsbereitschaft (Leimeister und David 2019a). Veränderungsbereitschaft wird dabei verstanden als Neugier und Offenheit gegenüber Neuem sowie einer daraus resultierenden Eigeninitiative und Lernmotivation zur Aktualisierung und Veränderung eigener Handlungsweisen und Wissensbestände (Hillebrand 2018).

Aufbauend auf den vorgestellten Erkenntnissen gehen wir davon aus, dass für die Facette Selbstkompetenz mindestens die Dimensionen Eigenverantwortung und Veränderungsbereitschaft benannt werden (Hypothese 5).

\subsubsection{Digitale Kompetenz in der digitalisierten Arbeitswelt}

Während die Begrifflichkeiten der zuvor beschriebenen Kompetenzfacetten einheitlich definiert sind, besteht bei digitalen Kompetenzen eine große Diversität von Begrifflichkeiten und Definitionen (Oberländer et al. 2020). Im englischen Sprachraum dominieren die Begriffe digital literacy und digital competence die wissenschaftliche Diskussion. 
Unter digital literacy wird dabei die grundsätzliche Fähigkeit verstanden, Informationen aus unterschiedlichen digitalen Quellen zu lesen, zu verstehen und zu nutzen (Bawden 2008; Gilster 1997). Damit handelt es sich um eine Weiterentwicklung des Alphabetisierungsgedankens (literacy), der grundsätzlich die Fähigkeiten zusammenfasst, relevante Informationen unabhängig von einem bestimmten Format oder Medium zu lesen, zu schreiben, zu verstehen und entsprechend zu nutzen. Diese Betrachtung geht über eine reduzierte Sichtweise von Fähigkeiten zum erfolgreichen Umgang mit digitalen Informationen hinaus (Bawden 2008). Digital competence wird definiert als „confident, critical and creative use of ICT [Information and Communication Technology] to achieve goals " (Ferrari 2012, S. 1). Die Ziele, die durch den Einsatz von Informationsund Kommunikationstechnologien erreicht werden sollen, können dabei in unterschiedlichen Arbeits- und Lebensbereichen liegen. Bei dieser Definition steht die konkrete Anwendung der Informations- und Kommunikationstechnologien stärker im Fokus als bei der Definition der digital literarcy. Im deutschsprachigen Raum finden sich neben dem Begriff digitale Kompetenzen auch die Begrifflichkeiten Digitalkompetenz (im Sinne einer Problemlösekompetenz im Zusammenhang mit digitalen Technologien; z. B. Wicht et al. 2018) oder IT-Kompetenz (im Sinne eines kompetenten Umgangs mit Informationstechnologien sowie Kenntnissen über die Technologien, den Umgang mit Daten, IT-Sicherheit und Datenschutz, z. B. Ulber und Remmers 2019).

Wir betrachten in unserem Kompetenzmodell digitale Kompetenzen im Arbeitskontext und orientieren uns im Folgenden an der Definition von Oberländer et al.: „Digital competencies at work are a set of basic knowledge, skills, abilities, and other characteristics that enable people at work to efficiently and successfully accomplish their job tasks regarding digital media at work. " (Oberländer et al. 2020, S. 5). Dabei handelt es sich um eine relativ breite Definition, die die Anwendung sämtlicher Informations- und Kommunikationstechnologien im Arbeitskontext beinhaltet.

In der Industrie 4.0 gewinnen durch die vermehrte Nutzung und Einbindung digitaler Technologien wie beispielsweise DWAS, vor allem Kompetenzen zur Anwendung und effektiven Nutzung dieser Technologien an Bedeutung. Dies betrifft den Umgang mit Hardware- und Softwarekomponenten (Acatech 2016; Hammermann und Stettes 2016; Oberländer et al. 2020). Dazu gehört auch ein generelles Verständnis über den Aufbau, die Funktionalitäten und die Funktionsprinzipien digitaler Systeme (Ostermeier et al. 2018). Die allgemeine Zunahme der Bedeutung dieser Kompetenzen ist in allen Fachbereichen und unabhängig von der Unternehmensgröße spürbar (Hammermann und Stettes 2016).
Auch die Kompetenz zur Informations- und Datenverarbeitung im Sinne einer gezielten und kritischen Nutzung des Internets zur Suche nach relevanten Inhalten wird in unterschiedlichen Studien als relevant identifiziert. Dazu gehören auch das Wissen und das Bewusstsein für Datenschutz und Datensicherheit im betrieblichen Kontext (Hammermann und Stettes 2016; Oberländer et al. 2020).

Bereits bei den Sozialkompetenzen wurde darauf hingewiesen, dass durch eine flexible Arbeitsorganisation kommunikative und kooperative Kompetenzen zukünftig an Bedeutung gewinnen. Unter Einbezug der digitalen Möglichkeiten, wie DWAS zur Abstimmung innerhalb des Teams oder über Teamgrenzen hinweg, kann es zusätzlich erforderlich werden, räumlich flexibel oder virtuell mit anderen Personen zusammenzuarbeiten oder die Arbeit zu organisieren (Kauffeld et al. 2016; Kauffeld und Maier 2020). In diesem Zusammenhang können neue Kompetenzen bei der digitalen Kommunikation und Kooperation, beispielsweise Kenntnisse zur guten und effektiven Gestaltung von Textund Sprachnachrichten, notwendig werden (Hammermann und Stettes 2016; Kauffeld et al. 2016; Oberländer et al. 2020).

Aufbauend auf den vorgestellten Erkenntnissen gehen wir davon aus, dass als relevante digitale Kompetenzen mindestens die Dimensionen IT-Anwendungskompetenz, Informations- und Datenverarbeitungskompetenz, Datenschutz und Datensicherheit sowie digitale Kommunikation und Kooperation benannt werden (Hypothese 6).

\subsection{Wege der Kompetenzentwicklung im betrieblichen Kontext}

Durch digitalisierte und automatisierte Prozessabläufe sowie die verstärkte Nutzung und Anwendung von DWAS verändern sich Arbeitsprozesse in der Produktion. Beschäftigte sehen sich mit veränderten und teilweise höheren Anforderungen konfrontiert. Die veränderten Anforderungen und der Wunsch von Unternehmen, die Beschäftigten flexibler und an unterschiedlichen Arbeitsstationen einsetzen zu können, führen dazu, dass zunehmend Weiterbildungen auch für an- und ungelernte Beschäftigte angeboten werden (Schöpper-Grabe und Vahlhaus 2019).

Der Aufbau der benötigten Kompetenzen kann, wie in Abb. 2 dargestellt, mit formalen, non-formalen oder informellen Lernprozessen erfolgen. Bisherige Erkenntnisse zu Kompetenzentwicklungsmaßnahmen für den Aufbau relevanter Kompetenzen in der Industrie 4.0 zeigen, dass Unternehmen vor allem formale Weiterbildungsformate einsetzen. Besonders relevant sind unternehmensinterne und unternehmensexterne Weiterbildungen (Acatech 2016). Bei der Zielgruppe der an- und ungelernten Beschäftigten findet Lernen vor allem non-formal als Unterweisung durch Kollegen oder Vorgesetzte statt. Lehrveranstaltungen oder das 
selbstgesteuerte Lernen mit Medien (z. B. Wikis oder interaktive Online-Lernprogramme) werden in der industriellen Praxis für an- und ungelernte Beschäftigte nur selten eingesetzt (Acatech 2016; Kortsch et al. 2019; Schöpper-Grabe und Vahlhaus 2019). Dennoch legen Studien nahe, dass die zunehmende Digitalisierung neue Chancen für betriebliches Lernen entstehen lässt (Dehnbostel 2019) und digitale Formate zur Kompetenzentwicklung relevanter werden (Kortsch et al. 2019; mbb Institut 2019). Mitarbeitende erhalten durch Digitalisierung und DWAS neue Möglichkeiten, um selbstständig neues Wissen aufzubauen (Kortsch et al. 2019; Leimeister und David 2019b). Dadurch könnte das selbstgesteuerte Lernen mit Medien als ein Weg nonformaler Kompetenzentwicklung in der Produktionsarbeit zukünftig an Bedeutung gewinnen.

Den vorgestellten Befunden folgend gehen wir davon aus, dass als Wege der formalen Kompetenzentwicklung mindestens interne und externe Kurse benannt werden (Hypothese 7). Bei non-formalen Wegen der Kompetenzentwicklung werden mindestens das Anlernen durch Kollegen und Vorgesetzte und das selbstgesteuerte Lernen mit digitalen Medien benannt (Hypothese 8).

\section{Methode}

In diesem Abschnitt werden die Datengewinnung und die Stichprobe vorgestellt. Weiterhin werden das Vorgehen bei der Datenaufbereitung und bei der Auswertung der qualitativen Daten beschrieben.

\subsection{Beschreibung des Interviewleitfadens}

Um zukünftig bedeutsame Kompetenzen für Mitarbeitende in der Produktion sowie Wege der Kompetenzentwicklung zu erfassen, wurden halbstrukturierte Interviews durchgeführt. Die Interviews beinhalteten fünf thematische Schwerpunkte: (1) Anwendungsmöglichkeiten von DWAS, (2) Veränderungen der Arbeitsaufgaben und -inhalte, (3) Zusammenarbeit und Führung, (4) Kompetenzen und Qualifizierung, (5) SWOT-Analyse. Der Interviewleitfaden bestand aus vorab formulierten Fragen, die im Rahmen des Interviews gestellt wurden. Die über alle Interviews festgelegte Struktur ermöglichte eine vergleichende Auswertung der Interviewdaten (Renner und Jacob 2020). Zusätzlich war es durch die Nutzung halbstrukturierter Interviews möglich, individuell auf den jeweiligen Interviewverlauf $\mathrm{zu}$ reagieren, die Reihenfolge von Fragen anzupassen und optional vertiefende Nachfragen zu stellen (Qu und Dumay 2011; Renner und Jacob 2020).

Für die vorliegende Studie waren die folgenden Leitfragen aus dem Themengebiet (4) Kompetenzen und Qualifizierung relevant:
- Welche Kompetenzen sind für Mitarbeitende in der Produktion im Jahr 2025 besonders wichtig?

- Wie erfolgt die Kompetenzentwicklung/Qualifizierung von Produktionsmitarbeitenden im Jahr 2025?

Die Interviewfragen wurden offen gestellt, ohne dass den Teilnehmenden Antwortmöglichkeiten vorgegeben wurden. Ziel war es, die Annahmen der einzelnen Interviewten möglichst breit zu erfassen und Einschränkungen oder Meinungsbildung durch präsentierte Antwortmöglichkeiten auszuschließen.

\subsection{Beschreibung der Stichprobe}

In den Interviews wurden die Sichtweisen unterschiedlicher Expert/innen aus der Wissenschaft, der Politik und der betrieblichen Praxis (Planer/innen, Betriebsrat, Mitarbeitende in der Produktion aus einem fertigenden Unternehmen mit variantenreicher Produktion) aufgenommen. Der Einbezug unterschiedlicher Blickwinkel bei der Ermittlung der notwendigen Kompetenzen führt bei der Ableitung zukünftig relevanter Kompetenzen zu einem realistischeren Gesamtbild (Klug 2011). Die Identifikation und Rekrutierung der relevanten Expertengruppen erfolgte anhand einer kriteriengeleiteten Auswahlstrategie (Akremi 2019; Okoli und Pawlowski 2004). Dem lag die Annahme zugrunde, dass die fünf Expertengruppen in unterschiedlicher Weise Einfluss auf die Verbreitung und Nutzung von DWAS im betrieblichen Kontext nehmen und damit Einfluss auf die Veränderung der Arbeitsgestaltung und die damit verbundenen Arbeitsanforderungen haben. So können Politische Akteure rechtliche Rahmenbedingungen für die betriebliche Nutzung von DWAS festlegen. Planer/innen können durch die Auswahl der jeweiligen Technologie, das Festlegen der Einsatzszenarien und die Einführung der DWAS in den Arbeitsprozess Einfluss auf die Gestaltung von Arbeit nehmen und dadurch veränderte Kompetenzanforderungen für Beschäftigte in der Produktion schaffen. Zur Bedeutung der einzelnen Expertengruppen sowie für eine Beschreibung zur Vorgehensweise bei der Identifikation und Rekrutierung siehe auch Blumberg und Kauffeld (2020).

In Summe wurden 76 Interviews von der Erstautorin des Artikels durchgeführt. Die Interviews waren Teil einer Delphi-Studie und dienten als qualitative Vorrunde. Bei der Konzeption der qualitativen Vorrunde wurde den Empfehlungen von Okoli und Pawlowski (2004) gefolgt, die beim Einbezug unterschiedlicher Expertengruppen zwischen 10 und 18 Interviews je Gruppe empfehlen.

Als demografische Variablen wurden das Alter der Befragten und die Berufserfahrung in Jahren erhoben. Beide Variablen wurden den datenschutzrechtlichen Bestimmungen des Unternehmens folgend kategorial erhoben. Die Verteilung der demografischen Variablen ist in Tab. 1 dar- 
Tab. 1 Beschreibung der Stichprobe

\begin{tabular}{|c|c|c|c|c|c|c|c|}
\hline \multicolumn{2}{|c|}{$\begin{array}{l}\text { Demografische } \\
\text { Variablen }\end{array}$} & \multirow{2}{*}{$\begin{array}{l}\text { Wissenschaft } \\
(n=16) \\
2\end{array}$} & \multirow{2}{*}{$\begin{array}{l}\text { Politische Akteure } \\
(n=14) \\
2\end{array}$} & \multirow{2}{*}{$\begin{array}{l}\text { Planer/in } \\
(n=17) \\
7\end{array}$} & \multirow{2}{*}{$\begin{array}{l}\text { Betriebsrat } \\
(n=14)\end{array}$} & \multirow{2}{*}{$\begin{array}{l}\text { Anwendende } \\
(n=15)\end{array}$} & \multirow{2}{*}{$\begin{array}{l}\text { Gesamt } \\
(n=76)\end{array}$} \\
\hline Alter (in & $21-30$ & & & & & & \\
\hline Jahren) & $31-40$ & 2 & 4 & 5 & 1 & 7 & 19 \\
\hline & $41-50$ & 4 & 4 & 4 & 6 & - & 18 \\
\hline & $51-60$ & 5 & 3 & 1 & 6 & 2 & 17 \\
\hline & $>60$ & 3 & 1 & - & - & - & 4 \\
\hline \multirow{5}{*}{$\begin{array}{l}\text { Berufs- } \\
\text { erfahrung } \\
\text { (in } \\
\text { Jahren) }\end{array}$} & $0-4$ & 1 & - & 6 & - & - & 7 \\
\hline & $5-9$ & - & 3 & 2 & 1 & 4 & 10 \\
\hline & $10-14$ & 3 & 3 & 2 & - & 3 & 11 \\
\hline & $15-19$ & 2 & - & 3 & - & 5 & 10 \\
\hline & $>19$ & 10 & 8 & 4 & 12 & 3 & 37 \\
\hline
\end{tabular}

Eine Person machte keine Angaben zum Alter und der Berufserfahrung

gestellt. Es fällt auf, dass die Altersverteilung der Teilnehmenden über vier der fünf Altersgruppen relativ ähnlich ist. Einzig die Altersgruppe $>60$ Jahre weist eine eher geringe Teilnehmerzahl auf $(n=4)$. In dieser Altersgruppe sind nur Teilnehmende der Gruppen Wissenschaft und Politische Akteure vorhanden. Ein Großteil der Interviewteilnehmenden gibt zudem an, über eine langjährige Berufserfahrung (>19 Jahre) zu verfügen.

\subsection{Datenaufbereitung und Vorgehen bei der Erstellung der Kategoriensysteme}

Die Aufbereitung der Interviewdaten erfolgte mit der Software MAXQDA (VERBI Software 2017). Die Transkription der Interviews erfolgte anhand der inhaltlich-semantischen Transkriptionsregeln (Dresing und Pehl 2018). Zur Aufbereitung und Strukturierung des Datenmaterials wurde die qualitative strukturierende Inhaltsanalyse (Steigleder 2008) angewendet.

Als Grundlage für die Entwicklung des Kategoriensystems zu Kompetenzen in der Industrie 4.0 dienten die vier Facetten Fach-, Methoden-, Sozial- und Selbstkompetenz (vgl. Kauffeld 2006; Kauffeld und Paulsen 2018; Sonntag und Schaper 1999). Aufgrund der besonderen Betrachtung digitaler Technologien im Produktionskontext wurde zusätzlich die Kategorie digitale Kompetenz als Querschnittskompetenz zu den vier bestehenden Kompetenzfacetten ergänzt. Die aus der Literatur abgeleiteten Kompetenzen wurden als erstes Grundgerüst für die Kodierung genutzt. Identifizierte Textabschnitte, die keiner dieser Kategorien zugeordnet werden konnten, wurden je Kompetenzfacette einer Kategorie Sonstiges zugeordnet. Vor einem zweiten Kodiervorgang wurden diese gesichtet, neue Kategorien herausgearbeitet und mit Erkenntnissen aus der Literatur ergänzt.

Für die Entwicklung des Kategoriensystems zu Wegen der Kompetenzentwicklung wurde, wie in Abb. 2 dargestellt, auf die Einteilung in formale, non-formale und informelle Kompetenzentwicklung zurückgegriffen (Kauffeld und Paulsen 2018; OECD 2007). Die aus der Literatur abgeleiteten Subkategorien dienten als erstes Grundgerüst bei der Kodierung. Auch bei der Entwicklung dieses Kategoriensystems wurden relevante Textabschnitte, die keiner bestehenden Kategorie zugeordnet werden konnten, zunächst der Kategorie Sonstiges zugeordnet. Vor einem zweiten Kodiervorgang wurde diese Kategorie gesondert betrachtet und unter Einbezug von Erkenntnissen aus der Literatur weitere Kategorien erstellt.

Zur Ermittlung der Güte des Kodierprozesses mit den vorliegenden Kategoriensystemen wurde die Intercoder-Reliabilität (Kappa, $\kappa_{n}$ Brennan und Prediger 1981) ermittelt. Nach einer ersten Kodierschulung wurden sechs zufällig ausgewählte Interviews von zwei Kodierern bearbeitet und für die kodierten Textstellen Kappa ermittelt. Für die kodierten Interviews wurde ein Kappa von $\kappa=0,83$ erreicht. Eine Übereinstimmung von $\kappa>0,80 \mathrm{kann}$ als starke Übereinstimmung bewertet werden (Rädiker und Kuckartz 2019).

\section{Ergebnisse}

In diesem Abschnitt werden die Ergebnisse der Befragung vorgestellt. Dazu wird zunächst das Kategoriensystem zu relevanten Kompetenzen für Produktionsbeschäftigte vorgestellt. Anschließend werden Unterschiede und Gemeinsamkeiten bei den Antworten der befragten Expertengruppen deskriptiv beschrieben. Abschließend werden die identifizierten Kategorien für Wege der Kompetenzentwicklung dargestellt und Unterschiede und Gemeinsamkeiten der befragen Expertengruppen vorgestellt.

\subsection{Kompetenzen für die Produktionsarbeit in der Industrie 4.0 mit DWAS}

Nachfolgend wird das erstellte Kompetenzmodell beschrieben und die formulierten Hypothesen überprüft. 


\subsubsection{Kompetenzmodell}

Als Ergebnis der Kodiervorgänge entstand ein positionsspezifisches Kompetenzmodell (vgl. Klug 2011), das spezifische Anforderungen an Produktionsbeschäftigte der Gewerke Montage und Logistik in der Industrie 4.0 und beim Umgang mit DWAS umfasst. Das Kompetenzmodell ist in Abb. 3 dargestellt.

In Summe wurden 18 Kompetenzen identifiziert. Davon sind sieben Kompetenzen der Querschnittskompetenz digitale Kompetenzen zugeordnet. Die identifizierten Kompetenzen konnten den etablierten Facetten der Fach-, Methoden-, Sozial- und Selbstkompetenz zugeordnet werden. Damit zeigt sich, dass auch im Zuge veränderter Anforderungen und einer zunehmenden Bedeutung digitaler Kompetenzen die Unterteilung in Fach-, Methoden-, Sozial- und Selbstkompetenz als Systematisierung für Kompetenzmodelle genutzt werden kann. Hypothese 1 kann damit nicht abgelehnt werden.

Für die Facette Fachkompetenz wurden Fachwissen, handwerkliche Fähigkeiten und Prozessdenken als relevante Kompetenzen identifiziert. Die gefundenen Kompetenzen stimmen mit den aus der Literatur abgeleiteten, bedeutsamen Kompetenzen für Produktionsbeschäftigte überein. Daher kann Hypothese 2 nicht abgelehnt werden.

Für die Facette Methodenkompetenz wurde, wie aus der Literatur abgeleitet, die Kompetenz Analysefähigkeit benannt. Zusätzlich wurde die Kompetenz Strategienflexibilität identifiziert. Bei Strategienflexibilität steht die Fähig- keit im Vordergrund, sich auf unterschiedliche nicht-routine Situationen einstellen $\mathrm{zu}$ können und unterschiedliche Strategien für deren Bewältigung zu entwickeln. Die erfolgreiche Bewältigung der nicht-routine Situationen macht es erforderlich, dass Beschäftigte unterschiedliches Wissen verknüpfen, reflektieren und auf die aktuelle Situation anpassen. Diese Anforderung wird auch im folgenden Zitat deutlich.

Also man programmiert einen Standardablauf so wie er sein sollte, das dauert so $5 \%$ der Zeit, und die restlichen $95 \%$ programmiert man Ausnahmen. Also was passiert, wenn etwas vorkommt, was eigentlich nicht vorgesehen war. [...] Und diese Liste dieser Ausnahmen [...] wird niemals vollständig sein. Und damit umzugehenlernen, erfordert ganz klar wesentlich mehr Kompetenz. Man muss ein bisschen Ahnung von Informatik haben, man muss den Herstellungsprozess begreifen, aber in erster Linie muss man das tun, was Menschen am besten können oder was sie besser können als Computer und das ist reflektiert zu handeln. Nicht Knöpfe drücken, sondern verstehen. (Interview 21, Planer/in)

Hypothese 3 kann daher nicht abgelehnt werden. Die aus der Literatur abgeleitete Kompetenz Analysefähigkeit kann um die Dimension Strategienflexibilität ergänzt werden.

Für die Facette Sozialkompetenz wurden die Kompetenzen Kommunikationsfähigkeit und Kooperationsfähigkeit benannt. Dies entspricht den aus der Literatur abgeleiteten

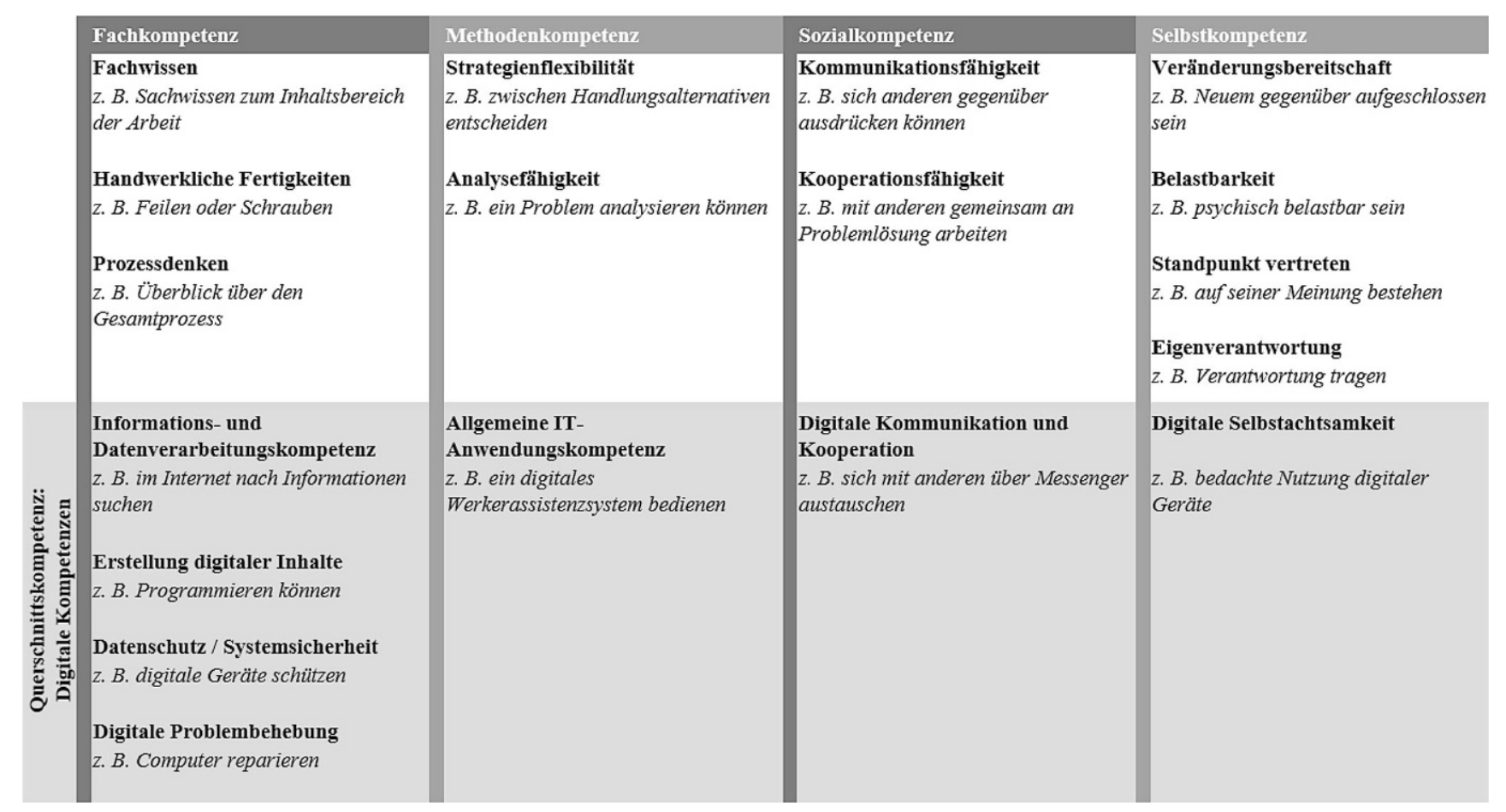

Abb. 3 Kategoriensystem Kompetenzen 
Kompetenzen. Hypothese 4 kann daher nicht abgelehnt werden.

Für die Facette Selbstkompetenz wurden die Kompetenzen Veränderungsbereitschaft und Eigenverantwortung aus der Literatur abgeleitet. Diese konnten durch die Daten aus den Interviews bestätigt werden. Darüber hinaus wurden die Kompetenzen Belastbarkeit und Standpunkt vertreten identifiziert.

Belastbarkeit ist die Fähigkeit, bei physischen und/oder psychischen Anforderungen der Arbeitsumwelt zielorientiert zu handeln und diese erfolgreich zu bewältigen. Anforderungen können aus den bestehenden Arbeitsbedingungen, der Arbeitsumwelt oder den sozialen Interaktionen (Konflikten) entstehen. Belastbarkeit beinhaltet auch einen positiven Umgang mit Risiken, Unbestimmtheiten und Widersprüchen sowie die Fähigkeit, daraus Ansätze für die Selbstentwicklung zu ziehen (Heyse 2007). Das nachfolgende Zitat zeigt, dass Effizienzsteigerung und Leistungsdruck neue Anforderungen sein können, mit denen die Beschäftigten umgehen können müssen.

Und wenn [...] natürlich dieses Schnelllebige dazukommt, dann wird das wieder für die [Anmerkung: für die Mitarbeitenden] auch zusätzlicher [...] Leistungsdruck [...]. Das ist der eine Punkt. Damit muss man schauen, dass man damit umgehen kann. (Interview 15, Planer/in)

Die Kompetenz Standpunkt vertreten beschreibt, dass Beschäftigte zukünftig vermehrt in der Lage sein müssen, ihren Standpunkt, ihre Meinung oder ihre Interessen in einem Diskurs mit anderen zu vertreten. Dies geht damit einher, dass Beschäftigte die Konsequenzen der Handlungen anderer erfassen, verstehen und reflektieren müssen sowie Auswirkungen daraus für die eigene Arbeitstätigkeit ableiten und eine Position beziehen müssen (Hillebrand 2018). Das bewusste Positionieren ist im folgenden Zitat erkennbar:

Dann entwickle ich meine eigenen Arbeitsprozesse weiter. Ich müsste auch ein Stück weit Politiker sein, ich müsste dann auch Einfluss darauf nehmen, in welche Richtung sich das entwickelt. (Interview 20, Politischer Akteur)

Hypothese 5 kann daher nicht abgelehnt werden. Neben den in der Literatur benannten Selbstkompetenzen Veränderungsbereitschaft und Eigenverantwortung konnten die Kompetenzen Belastbarkeit und Standpunkt vertreten identifiziert werden.

Für die digitale Kompetenz wurden sieben relevante Kompetenzdimensionen ermittelt, die ebenfalls den vier Facetten Fach-, Methoden-, Sozial- und Selbstkompetenz zugeordnet werden konnten. Damit zeigt sich, dass digitale Kompetenz als Querschnittskompetenz beschrieben werden kann. Eine Querschnittskompetenz kann verstanden werden als eine Kompetenz, die sich aus mehreren unterschiedlichen Kompetenzen zusammensetzt (Erpenbeck 2012). Wie aus der Literatur abgeleitet, konnten die Kompetenzen Informations- und Datenverarbeitungskompetenz, Datenschutz/Systemsicherheit, Allgemeine IT-Anwendungskompetenz und Digitale Kommunikation und Kooperation identifiziert werden. Darüber hinaus wurden die Kompetenzen Erstellung digitaler Inhalte, digitale Problembehebung und digitale Selbstachtsamkeit identifiziert.

Die Kompetenz Erstellung digitaler Inhalte knüpft an die dargestellte Definition der digital literacy an. Hier wird explizit auf das Schreiben bzw. das Erstellen von Inhalten in digitalen Arbeitskontexten eingegangen. Dies umfasst sowohl das Aufschreiben und Bereitstellen von Informationen zum Arbeitsprozess oder -ablauf, wie es im nachfolgenden Zitat dargestellt wird, als auch rudimentäre Programmierkenntnisse. Damit kommt den Produktionsbeschäftigten keinesfalls nur ein passiver Part im Umgang mit den dargestellten und bereitgestellten Informationen durch DWAS zu. Vielmehr unterstreicht es die Anforderung an Beschäftigte, sich selbst aktiv in die Erstellung von Inhalten, das Teilen von Wissen und Informationen sowie die Gestaltung des Unternehmens einzubringen.

Und über solche zum Beispiel Unternehmenswikis oder Social Media-Formen im Unternehmen: Da sind natürlich nicht nur die Leitungsebenen gefordert, da was einzustellen, sondern in gleichem Maße stellen auch die Mitarbeiter was ein, zum Beispiel was sie gerade bemerken im Bereich der Montage oder was sie bemerken im Bereich der Produktion, der Qualität der Werkzeuge, der Qualität der Vorprodukte oder des häufigen Wechsels oder den Ausfällen von Maschinen oder die gute Laufzeit von einer bestimmten Maschine. (Interview 4, Politischer Akteur)

Bei der Kompetenz digitale Problembehebung wird die dargestellte Definition zur Digitalkompetenz aufgegriffen, in der vor allem die Problemlösung im Zusammenhang mit digitalen Technologien betrachtet wird (Wicht et al. 2018). Zur digitalen Problembehebung zählen beispielswiese das Reparieren eines technischen Gerätes oder das Aufladen eines Akkus, aber auch die Fähigkeit mit angezeigten Fehlermeldungen umzugehen.

Ich sage einfach mal, wir möchten was einführen und du gehst zum Beispiel eine Woche auf eine Schulung, wo du mit diesen Systemen Vor- und Nachteile und einwandfreies Arbeiten anwenden kannst, inklusive der verschiedensten Möglichkeiten, natürlich auch Anwenderfehler oder Systemprobleme selbst beheben können. Ich sage mal, die Tendenz geht da hin in Richtung Digitalisierung und Systeme und Rechner [...]. Das heißt natürlich, wenn irgendwo dann Pro- 
bleme sein sollten, müsste der Mitarbeiter am besten diese auch selbst [...] lösen können. (Interview 43, Anwendende)

Weiterhin wurde die Kompetenz digitale Selbstachtsamkeit aus den Interviews heraus identifiziert. Digitale Selbstachtsamkeit wird definiert als die Fähigkeit bei der Anwendung und Nutzung digitaler Technologien im Arbeitskontext Risiken zu erkennen und entsprechende Konsequenzen abzuleiten, um die eigene physische und psychische Gesundheit und das eigene Wohlbefinden zu schützen. Dies wird in den folgenden Zitaten verdeutlicht.

Wenn die mobilen Endgeräte oder auch Wearables dann zum Einsatz kommen, dann müssen die Mitarbeiter dafür auch sensibilisiert werden, wann sie das selbst [..] ausschalten. [...] Ja, also die müssen eine Befähigung entwickeln, selber, und da kann der Arbeitgeber dann tatsächlich auch unterstützen, dass sie diese Entgrenzung zwischen Privatleben und Arbeitsleben selbst vornehmen können und dazu auch ermächtigt werden, das auch durchzusetzen. (Interview 9, Politischer Akteur)
Warum lerne ich nicht damit umzugehen, und zwar so in Maßen, dass es mir nicht schadet? (Interview 46, Betriebsrat)

Neben den aus der Literatur abgeleiteten Kompetenzen Informations- und Datenverarbeitungskompetenz, Datenschutz/Systemsicherheit, allgemeine IT-Anwendungskompetenz und digitale Kommunikation und Kooperation konnten außerdem die Kompetenzen Erstellung digitaler Inhalte, digitale Problembehebung und digitale Selbstachtsamkeit generiert werden. Hypothese 6 kann daher nicht abgelehnt werden.

\subsubsection{Deskriptive Gruppenvergleiche für Kompetenzen in der Industrie 4.0}

Bei der Nennung der einzelnen Kompetenzen sind Unterschiede zwischen den fünf befragten Expertengruppen erkennbar. In Abb. 4 ist die Verteilung der Nennhäufigkeiten für die Kompetenzen aus den Facetten Fach-, Methoden-, Sozial- und Selbstkompetenz dargestellt. Abgetragen ist die prozentuale Häufigkeit der Nennungen einer bestimmten Kompetenz je Expertengruppe. Mehrfachnennungen derselben Kompetenz in einem Interview wurden nur einmal

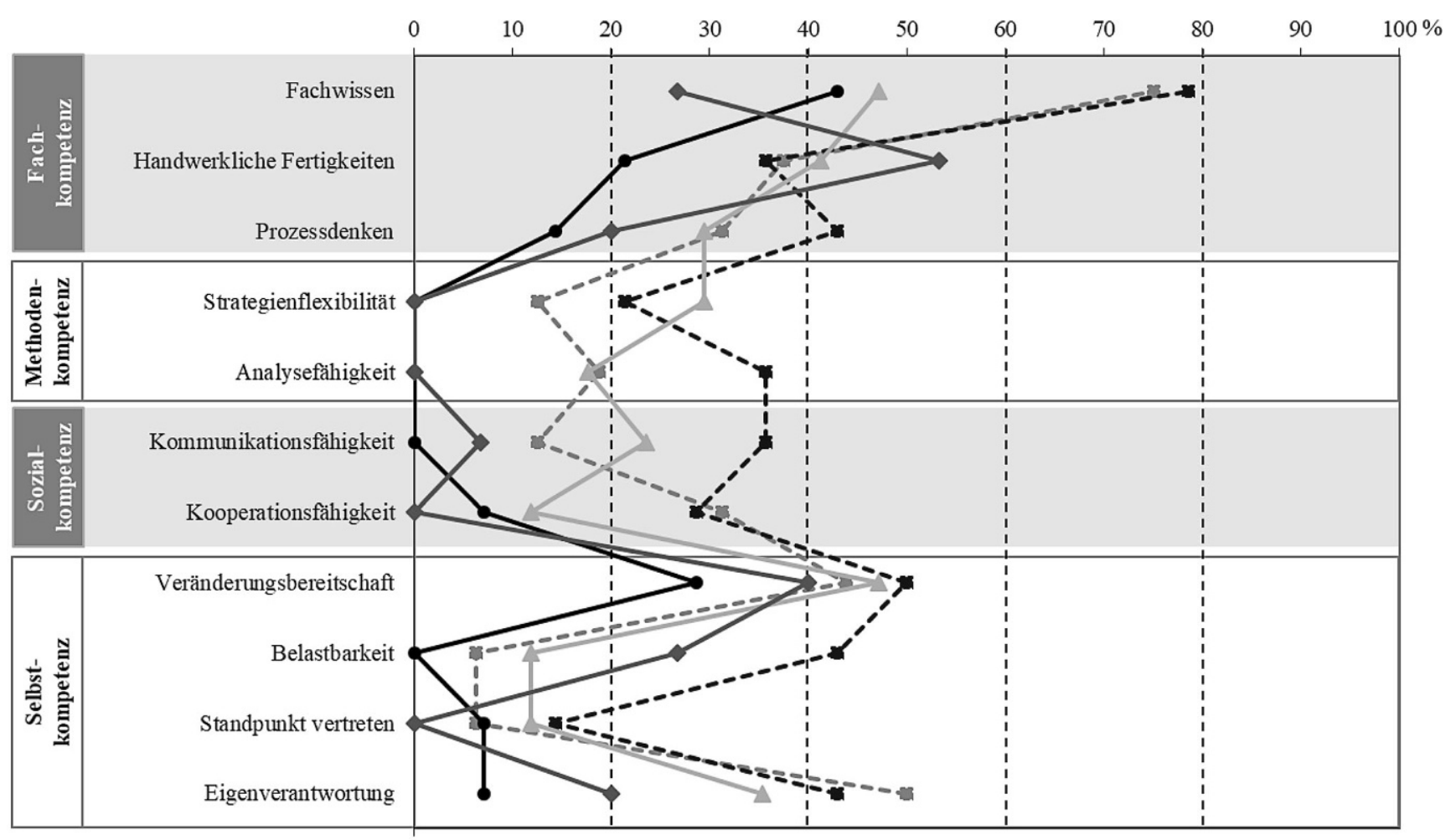

-- Wissenschaft $(n=16)-\infty$ - Politische Akteure $(n=14) \longrightarrow$ Planer/in $(n=17) \longrightarrow$ Betriebsrat $(n=14) \longrightarrow$ Anwendende $(n=15)$

Abb. 4 Deskriptive Auswertung Nennungen je Expertengruppe für relevante Fach-, Methoden-, Sozial- und Selbstkompetenz 


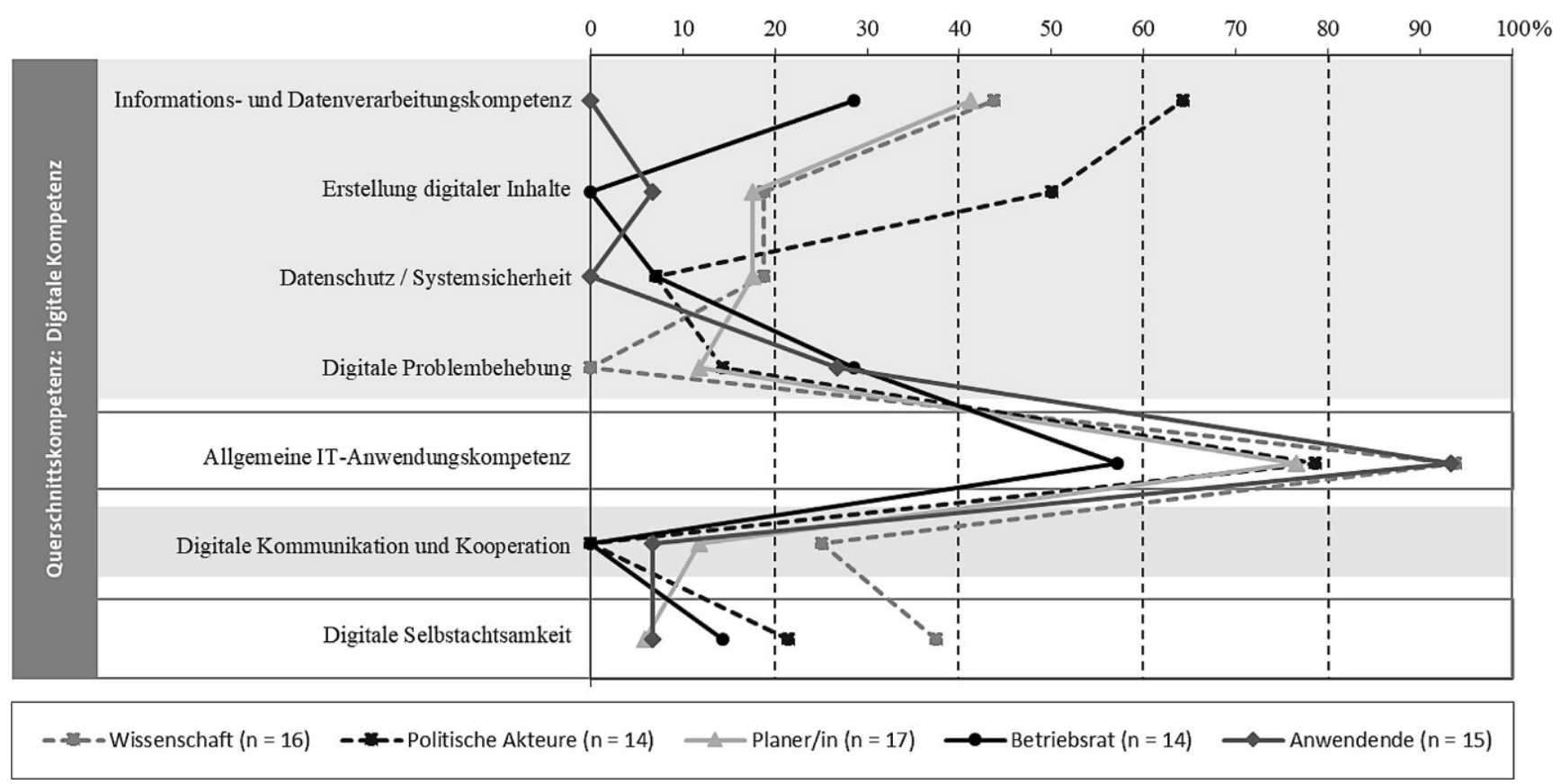

Abb. 5 Deskriptive Auswertung Nennungen je Expertengruppe für relevante digitale Kompetenzen (Hinweis: Bei der Kompetenzdimension Datenschutz/Systemsicherheit liegen die Datenpunkte für die Gruppen Politische Akteure und Betriebsrat aufeinander (mit jeweils 2 Nennungen))

gezählt. Somit bedeutet ein Wert von $100 \%$, dass eine bestimmte Kompetenz von allen Interviewteilnehmenden einer bestimmten Expertengruppe benannt wurde, $0 \%$ bedeutet, dass die jeweilige Kompetenz von einer bestimmen Expertengruppe gar nicht benannt wurde.

In der Facette Fachkompetenz fällt auf, dass die Bedeutung des Fachwissens vor allem von Interviewteilnehmenden der Expertengruppen Politische Akteure und Wissenschaft benannt wurde. In beiden Gruppen wurde das Fachwissen als relevante Kompetenz in mehr als $70 \%$ der Interviews benannt (Wissenschaft: $75 \%$, Politische Akteure: $79 \%$ ). In allen drei Expertengruppen der betrieblichen Praxis wird diese Kompetenz in weniger als $50 \%$ der Interviews benannt. Beim Blick auf die Facette Methodenkompetenz wird deutlich, dass Kompetenzen aus dieser Facette insgesamt weniger häufig und ausschließlich von den Expertengruppen Politische Akteure, Wissenschaft und Planer/in genannt wurden. Teilnehmende der beiden Gruppen Betriebsrat und Anwendende benennen weder Strategienflexibilität noch Analysefähigkeit als relevante Kompetenz. In der Facette Sozialkompetenz weisen die beiden Dimensionen Kommunikationsfähigkeit und Kooperationsfähigkeit eine große Streuung auf. Die Dimension Kommunikationsfähigkeit wurde von Betriebsräten gar nicht benannt, wohingegen Politische Akteure diese Kompetenz in 5 Interviews (36\%) benannten. Die Dimension Kooperationsfähigkeit wurde von der Gruppe der Anwendenden gar nicht genannt. In der Facette Selbstkompetenz werden vor allem die Aspekte Veränderungsbereitschaft, Belastbarkeit und Eigenverantwortung benannt. Die Kompetenz eigenen Standpunkt vertreten wird von den Expertengruppen Wissenschaft, Politische Akteure, Betriebsrat und Planer/in selten und von Anwendenden gar nicht benannt.

In Abb. 5 sind die deskriptiven Vergleiche der prozentualen Nennhäufigkeiten je Expertengruppe für die identifizierten digitalen Kompetenzen dargestellt. $100 \%$ bedeutet, dass eine bestimmte Kompetenz von allen Interviewteilnehmenden einer bestimmten Expertengruppe benannt wurde, $0 \%$ bedeutet, dass die jeweilige Kompetenz von einer bestimmen Expertengruppe gar nicht benannt wurde. Mehrfachnennungen derselben Kompetenz in einem Interview gingen nur einmal in die Nennhäufigkeit ein.

Bei den digitalen Kompetenzen, die der Facette Fachkompetenz zugeordnet sind, fällt auf, dass vor allem die Informations- und Datenverarbeitungskompetenz über die Expertengruppen hinweg sehr unterschiedlich häufig benannt wird. Während diese Kompetenz von Anwendenden gar nicht benannt wird, wird die Kompetenz von der Gruppe der Politischen Akteure in 9 Interviews (64\%) benannt. Die allgemeine IT-Anwendungskompetenz, die der Facette der Methodenkompetenz zugeordnet wurde, wird über alle Kompetenzdimensionen hinweg am häufigsten als notwendige Kompetenz benannt. Die digitale Kommunikation und Kooperation wird nur von den Expertengruppen Wissenschaft, Planer/in und Anwendende benannt. Die digitale Selbstachtsamkeit wird insgesamt selten, jedoch von allen Expertengruppen benannt. 


\subsection{Wege der Kompetenzentwicklung in der Industrie $\mathbf{4 . 0}$}

Wie können Kompetenzentwicklungswege beschrieben werden? Im Folgenden werden unterschiedliche Zugänge anhand des erstellten Kategoriensystems beschrieben, die von den Expertengruppen (Wissenschaft, Politische Akteure, Planer/in, Betriebsräte und Anwendende) unterschiedlich häufig benannt wurden.

\subsubsection{Kategoriensystem für Wege der Kompetenzentwicklung}

Insgesamt konnten acht unterschiedliche Wege der Kompetenzentwicklung identifiziert werden. Diese konnten anhand des bestehenden Ordnungsrahmens in formale, non-formale und informelle Kompetenzentwicklung unterschieden werden (siehe Abb. 6).

Als Wege der formalen Kompetenzentwicklung wurden in den Interviews, wie aus der Literatur abgeleitet, betriebsexterne und betriebsinterne Kurse benannt. Damit kann Hypothese 7 nicht abgelehnt werden.

Als non-formale Kompetenzentwicklungsmaßnahmen wurden sowohl das Anlernen durch Kollegen als auch das selbstgesteuerte Lernen mit Medien benannt. Weiterhin wurden in den Interviews zusätzlich das digitale Anlernen, Unterweisen und Informieren sowie Lernfabriken als Möglichkeiten zur non-formalen Kompetenzentwicklung benannt.

Die Kategorie digitales Anlernen, Unterweisen und Informieren beschreibt die Nutzung digitaler Endgeräte für die Sensibilisierung von Mitarbeitenden zu bestimmten Themen. Hierbei steht die Informationsvermittlung durch die digitalen Endgeräte im Fokus. Die Kategorie unterscheidet sich von der Kategorie selbstgesteuertes Lernen mit Medien durch einen stärker verpflichtenden Charakter und den Lernort, der sich in unmittelbarer Nähe zum Arbeitsplatz befindet. Die Kategorie selbstgesteuertes Lernen mit Medien wurde ausschließlich dann vergeben, wenn die Interviewteilnehmenden herausstellten, dass die Mitarbeitenden selbst entscheiden können, ob, wann und wo sie die Kompetenzentwicklung durchführen wollen.

Weiterhin wurde die Lernfabrik als ein Weg non-formaler Kompetenzentwicklung benannt. Eine Lernfabrik wird definiert als eine Lernumwelt, die authentische Arbeitsprozesse nachstellt, unterschiedliche Arbeitsstationen beinhaltet und dabei sowohl technische als auch organisationale Aspekte der realen Arbeitssituation abbildet. Sie zeichnet sich durch ein veränderbares Setting aus, in dem physische Produkte erstellt werden. Nicht nur reale physische Räume, sondern auch virtuelle Abbildungen der realistischen Arbeitsprozesse können als Lernfabriken (im weiteren Sinne) verstanden werden (Abele et al. 2017, 2016). Daher wurden dieser Kategorie sowohl Nennungen von Modellfabriken, Experimentier-Inseln und Musterarbeitsplätzen als auch virtuelle Simulationen der realen Arbeitsumgebung zugeordnet. Das nachfolgende Zitat beschreibt eine solche virtuelle Lernfabrik.

Um mal ganz niederschwellig anzufangen, also könnten das so virtuelle Lerngelegenheiten sein, [...] so virtuelle Lernszenarien. Das heißt, man bildet einen Produktionsprozess digital ab, zum Beispiel mittels Virtual Reality, was nicht immer heißt, dass man so eine voll immersive Brille braucht, man kann sich das genauso gut auch am Desktop anschauen und kann da eben aufgabenbezogen Aufgaben lösen. [...] Und die Maschine, die virtuelle, verhält sich dann eben genau wie die reale. (Interview 59, Wissenschaft)

Als non-formale Maßnahmen der Kompetenzentwicklung wurden, wie aus der Literatur abgleitet, Anlernen, Unterweisung und Information durch Kollegen und das selbst-

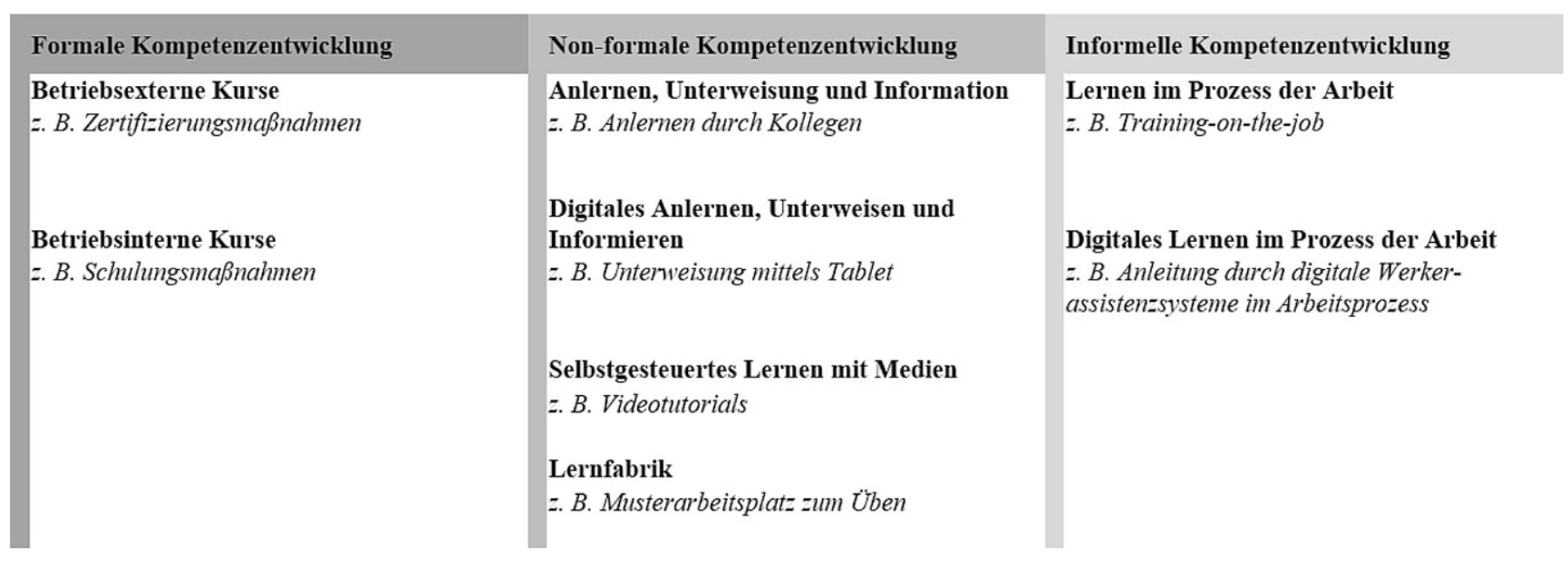

Abb. 6 Kategoriensystem Wege der Kompetenzentwicklung 
gesteuerte Lernen mit Medien identifiziert. Weiterhin wurden das digitale Anlernen, Unterweisen und Informieren sowie der Kompetenzaufbau mittels Lernfabriken identifiziert. Damit kann Hypothese 8 nicht abgelehnt werden.

Als informelle Wege der Kompetenzentwicklung wurden das Lernen im Prozess der Arbeit und das digitale Lernen im Prozess der Arbeit identifiziert. Lernen im Prozess der Arbeit wird auch als arbeitsintegriertes Lernen bezeichnet und als Teil des Arbeitshandelns verstanden. Der Kompetenzaufbau erfolgt dabei eher zufällig (Dehnbostel 2019). Hier wurden Nennungen wie Training-on-the-job, Job Enrichment, Job Enlargement sowie der informelle Austausch mit Kollegen während der Arbeit aufgenommen. Analog dazu beschreibt das digitale Lernen im Prozess der Arbeit, dass Beschäftigte durch die Verwendung von DWAS im Prozess der Arbeit relevante Kompetenzen für die Erfüllung der Arbeitsaufgabe aufbauen. Dies wird im folgenden Zitat dargestellt:

Also [...] bei so einer Smartwatch, vielleicht wird ja dann auch schon der Arbeitsprozess entsprechend dort eingeblendet und es steht schon alles digital darauf und man drückt nur noch auf Start und auf einmal geht es los. Oder [...] man geht von Prozessschritt zu Prozessschritt [...]. Ein Beispiel: [...] die Nachbestellreferenz auf einer Nachbestellkarte soll eingescannt werden. Das sagt dann einem vielleicht nicht mehr der Teamsprecher oder der Kollege, sondern das sagt einem diese Smartwatch, dieses Assistenzsystem. (Interview 47, Anwendende)

\subsubsection{Deskriptive Gruppenvergleiche für Wege der Kompetenzentwicklung}

Auch bei der Benennung von Lernwegen gibt es Unterschiede zwischen den einzelnen Expertengruppen. Die Verteilung der Nennhäufigkeiten je Expertengruppe ist in Abb. 7 dargestellt. Abgetragen ist die prozentuale Häufigkeit der Nennungen zu Lernwegen je Expertengruppe. Mehrfachnennungen des gleichen Lernweges in einem Interview wurden nur einmal gezählt. Somit bedeutet ein Wert von $100 \%$, dass ein bestimmter Lernweg von allen Interviewteilnehmenden einer bestimmten Expertengruppe benannt wurde, $0 \%$ bedeutet, dass der jeweilige Weg der Kompetenzentwicklung von einer bestimmen Expertengruppe gar nicht benannt wurde.

Bei der Betrachtung der formalen Wege der Kompetenzentwicklung fällt auf, dass vor allem betriebsexterne Kurse über alle Expertengruppen hinweg sehr selten genannt werden. Dafür zeigt sich, dass betriebsinterne Kurse als relevante Möglichkeit beim Aufbau der benötigten Kompetenzen für die Industrie 4.0 bei Produktionsbeschäftigten gesehen werden. Alle Expertengruppen benennen diese Möglichkeit. Die Wege der non-formalen Kompetenzentwicklung werden ebenfalls von allen Expertengruppen benannt. Beim digitalen Anlernen, Unterweisen und Informieren zeigt sich, dass Anwendende diese Form der Kompetenzentwicklung deutlich seltener benennen als die anderen Expertengruppen. Das selbstgesteuerte Lernen mit Medien wird vor allem von Planer/innen, Betriebsräten und Politischen Akteuren benannt. Auch der Aufbau relevanter

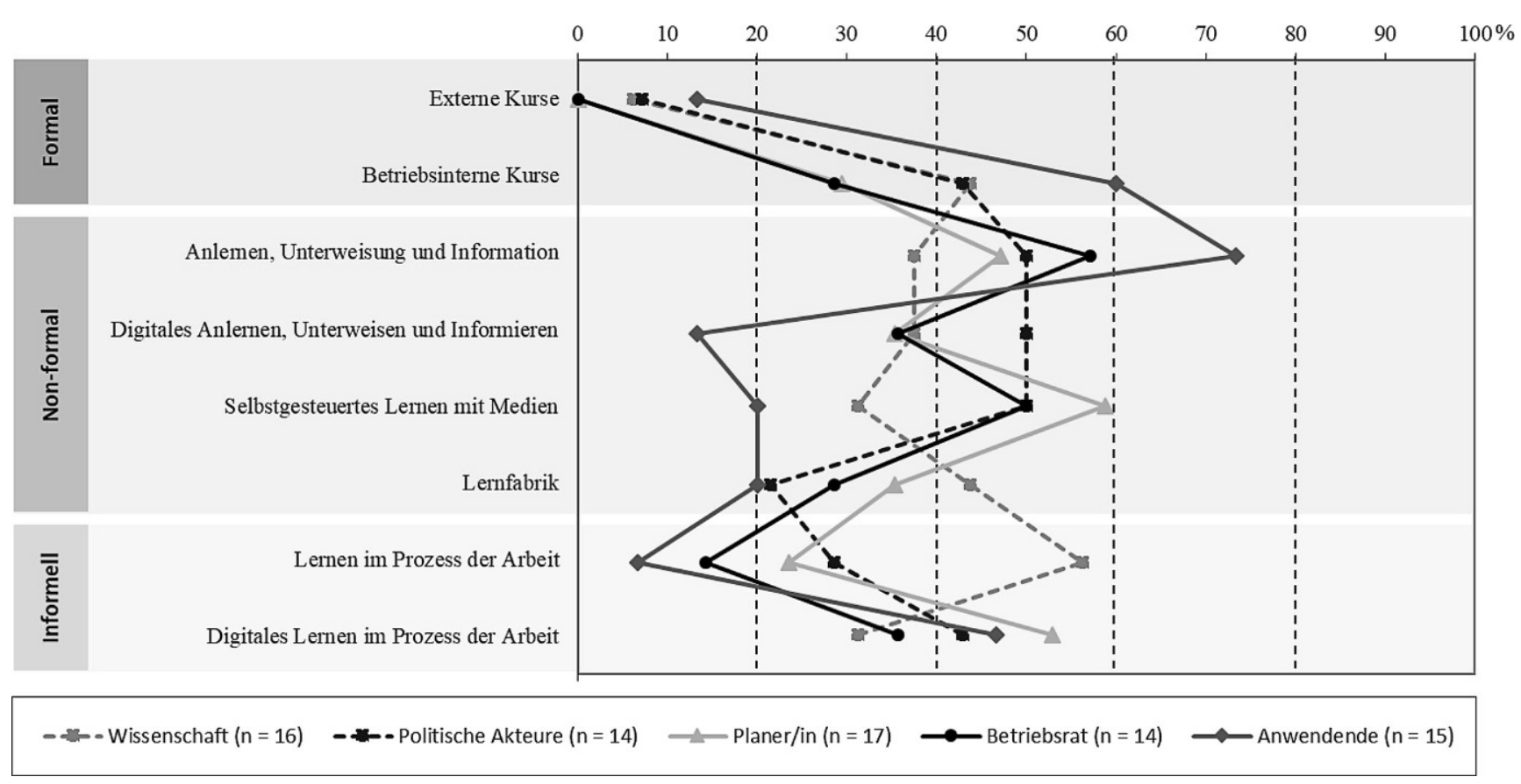

Abb. 7 Deskriptive Auswertung Nennungen je Expertengruppe für Wege der Kompetenzentwicklung 
Kompetenzen in einer Lernfabrik wird über alle Expertengruppen eher selten benannt. Bei der Betrachtung der informellen Wege der Kompetenzentwicklung wird deutlich, dass das Lernen im Prozess der Arbeit vor allem von Teilnehmenden aus dem Cluster Wissenschaft benannt wird. Das digitale Lernen im Prozess der Arbeit wird über alle Expertengruppen hinweg ähnlich häufig genannt.

\section{Diskussion}

Ziel dieser Studie war es, notwendige Kompetenzen sowie Wege der Kompetenzentwicklung für Einfachbeschäftigte in der Produktion (v. a. in den Gewerken Montage und Logistik) bei vermehrter Anwendung von DWAS zu ermitteln und ein Kompetenzmodell zu erstellen. Nachfolgend werden die Ergebnisse zusammengefasst und eingeordnet.

\subsection{Die Relevanz von digitalen Kompetenzen nimmt zu}

Als Ergebnis der 76 Interviews mit Experten aus der Wissenschaft, der Politik sowie der betrieblichen Praxis (Planer/in, Betriebsräte und Anwendende) konnte ein Kompetenzmodell für Produktionsbeschäftigte in der Industrie 4.0 mit 18 Dimensionen entwickelt werden. Die identifizierten Kompetenzen lassen sich den etablierten Kompetenzfacetten Fach-, Methoden-, Sozial- und Selbstkompetenz zuordnen (vgl. Abb. 3). Die aus der Literatur abgeleiteten Kompetenzen konnten in den Interviews bestätigt und durch die Methodenkompetenz Strategienflexibilität, die Selbstkompetenzen Belastbarkeit und Standpunkt vertreten sowie die digitalen Kompetenzen Erstellung digitaler Inhalte, digitale Problembehebung und digitale Selbstachtsamkeit ergänzt werden.

Die zunehmende Bedeutung digitaler Kompetenzen auch in der einfachen Produktionsarbeit ist daran $\mathrm{zu}$ erkennen, dass für die Facette digitale Kompetenz sieben Kompetenzen ermittelt werden konnten. Damit bestätigen die Ergebnisse frühere Studien, die gezeigt haben, dass digitale Kompetenzen berufsübergreifend an Bedeutung gewinnen (Hammermann und Stettes 2016; Pfeiffer et al. 2016). Die sieben Kompetenzen lassen sich anhand der Facetten Fach-, Methoden-, Sozial- und Selbstkompetenz systematisieren. Bereits bei der Durchsicht der Literatur fiel auf, dass es sich bei digitaler Kompetenz keinesfalls um ein eindimensionales Konstrukt handelt, sondern der Begriff übergreifend und im Sinne eines Sammelbegriffs genutzt wird. Damit kann digitale Kompetenz als Querschnittskompetenz verstanden werden (Erpenbeck 2012), die aus diversen Aspekten besteht (vgl. Ferrari 2012; Oberländer et al. 2020).

In der vorliegenden Studie wird vor allem die digitale Kompetenz zur Bedienung und zielorientierten Anwen- dung digitaler Technologien, im Sinne einer allgemeinen IT-Anwendungskompetenz, über alle Expertengruppen hinweg häufig benannt. Nutzt man die Nennhäufigkeit als Anzeichen für die Relevanz (Gläser und Laudel 2010; Kuckartz 2016; Saracevic 2006), bedeutet dies, dass es sich bei der allgemeinen IT-Anwendungskompetenz um eine über alle Expertengruppen hinweg als besonders relevant eingeschätzte Kompetenz handelt. Dieses Ergebnis könnte angesichts der weiten Verbreitung und sicheren Bedienung digitaler Endgeräte in privaten Lebensbereichen (Bär et al. 2020) verwundern, da grundlegende Kompetenzen bei der Anwendung digitaler Technologien somit vorausgesetzt werden könnten. Dennoch unterscheiden sich industrielle Anwendungen von DWAS vielfach von der privaten Nutzung (Hammermann und Stettes 2016) und machen daher einen gezielten Aufbau der Bedien- und Anwendungskompetenz bei Anwendenden erforderlich.

Unterschiedliche Sichtweisen der Expertengruppen ergeben sich vor allem für die Dimensionen Fachwissen, Belastbarkeit, Eigenverantwortung sowie für die digitalen Kompetenzdimensionen Informations- und Datenverarbeitungskompetenz, Erstellung digitaler Inhalte, digitale Problembehebung und digitale Kommunikation und Kooperation. Bei der Dimension Fachwissen fällt auf, dass diese Anforderung vor allem von den beiden externen Expertengruppen, den Wissenschaftler/innen und Politischen Akteuren, benannt wird. Teilnehmende aus den Gruppen der betrieblichen Praxis benannten diese Dimension wesentlich seltener. Grund dafür könnte sein, dass die Teilnehmenden aus der betrieblichen Praxis einen direkteren Bezug zu den Arbeitsplätzen und Arbeitsprozessen im betrieblichen Kontext haben. Die Arbeitsschritte in der industriellen Produktion sind häufig stark standardisiert (Bouville und Alis 2014) und können durch einfaches Anlernen und Training vermittelt werden (Hirsch-Kreinsen 2017). Dieser Umstand könnte bei den Teilnehmenden aus der betrieblichen Praxis bei der Beantwortung der Fragen präsenter gewesen sein. Durch den zukünftig stärkeren Einsatz von DWAS könnte zudem das notwendige Fachwissen kontextsensibel und bedarfsgerecht zur Verfügung gestellt oder abgerufen werden, sodass Fachwissen als weniger relevant wahrgenommen und daher weniger häufig benannt wurde. Dieser Aspekt wird auch im folgenden Zitat deutlich:

Vielleicht ist es gar nicht mehr notwendig, dass ich Wissen in einem großen Umfang zur Verfügung haben muss, weil ich auf dieses Wissen ganz anders zurückgreifen kann. (Interview 56, Wissenschaft)

Die hier dargelegte Annahme ist jedoch vermutlich ausschließlich für stark standardisierte, repetitive und routinebasierte Tätigkeiten haltbar. Dies wird auch in folgendem Zitat deutlich: 
Natürlich kann man auch da wieder ketzerisch sagen: Ich kann über diese Medien Schritt-für-SchrittAnleitungen anzeigen, sodass eigentlich auch jeder Ungelernte eine Tätigkeit ausführen kann, wie heute Spielzeug zusammenbauen. Aber so einfach wird es vermutlich nicht sein. Weil wenn sich kleinste Änderungen ergeben, die vielleicht nicht richtig dokumentiert sind, muss ich auch in der Lage sein, sowas durch ein gewisses technisches Verständnis zu hinterfragen. Alleine auch um Qualitätsfehler zu vermeiden. (Interview 61, Politischer Akteur)

Ein weiterer Grund für den extremen Unterschied bei der Nennhäufigkeit der Dimension Fachwissen könnte darin liegen, dass Anwendende ggf. stärker die Durchführung des Arbeitsschrittes (z. B. das richtige Montieren und Verschrauben von Produktbestandteilen) bei der Beschreibung relevanter Kompetenzen in den Fokus stellten. Dieser Aspekt wurde in der Dimension handwerkliche Fähigkeiten erfasst. Wissenschaftler/innen und Politische Akteure stellten hingegen stärker das notwendige Wissen über den richtigen Verbau in den Fokus, was der Dimension Fachwissen zugeordnet wurde.

Auch bei der Kompetenzdimension Informations- und Datenverarbeitungskompetenz unterscheiden sich die Nennhäufigkeiten zwischen den Expertengruppen stark. Während die Kompetenz von Anwendenden gar nicht benannt wurde, wurde die Kompetenz von Politischen Akteuren in $64 \%$ der Interviews benannt. Die verstärkte Anforderung an Mitarbeitende, Informationen und Daten $\mathrm{zu}$ suchen, kritisch zu bewerten und für die eigene Arbeit zu nutzen, könnte ein Indiz für die zunehmende Komplexität und die Zunahme von nicht-routine Tätigkeiten in der Einfacharbeit sein. Dies passt zu der Einschätzung, dass die zunehmende Anwendung digitaler Technologien im Produktionsumfeld zu einem Upgrading aller Tätigkeiten und somit auch der Einfacharbeit führen könnte (vgl. Hirsch-Kreinsen 2016). Upgrading beschreibt, dass mit dem Einsatz digitaler Technologien im Arbeitskontext eine Aufwertung von Tätigkeiten durch höhere Arbeitsanforderungen einhergeht (HirschKreinsen 2016). Mit der Anforderung der Informationsund Datenverarbeitungskompetenz werden Mitarbeitende aktiv in die Pflicht genommen, sich kritisch und unter Zuhilfenahme von digitalen Technologien an der Lösung von nicht-routine Aufgaben $\mathrm{zu}$ beteiligen. Auch in der benannten Kompetenz digitale Inhalte erstellen könnte ein stärkerer Wunsch nach der aktiven Partizipation und Mitgestaltung der Arbeit durch die Produktionsbeschäftigten sichtbar werden, der sich vor allem in den Antworten von Planer/innen sowie Wissenschaftler/innen und Politischen Akteuren zeigt. Beide Kategorien werden von Anwendenden selbst jedoch gar nicht oder kaum benannt. Eine Ursache hierfür könnte in der vorwiegend taktgebundenen
Arbeit in der Produktion bestehen. Bei einer zeitlichen Taktung von Arbeit, wie sie im betrachteten produzierenden Unternehmen heute an vielen Stellen vorzufinden ist, besteht aus Sicht der Anwendenden ggf. nicht ausreichend Zeit, um unter Verwendung von DWAS nach Lösungen für Probleme zu suchen.

Anders zeigt sich das Antwortverhalten für die digitale Kompetenz digitale Problembehebung. Diese wird vorwiegend von Anwendenden und Betriebsräten benannt und könnte ebenso als Indiz für ein Upgrading der Produktionsarbeit gewertet werden. Das nachfolgende Zitat eines Interviewteilnehmenden aus der Gruppe der Anwendenden zeigt, dass diese Kompetenz zwar mit dem Wunsch nach einer verantwortungsvolleren und bedeutsameren Tätigkeit einhergeht, jedoch gleichzeitig den Wunsch nach einem reibungslosen Ablauf der Arbeitsprozesse widerspiegelt.

Er (Anmerkung: Der Mitarbeitende) muss also mit dem Händlingsgerät, was er da bei sich hat, egal welches, müsste er selbstständig umgehen können und selbstständig auch diese Fehlermeldungen oder, wenn da irgendwas ist, Störungen beseitigen können. Das ist mehr Verantwortung für den Mitarbeiter [...]. Das ist gut für den Menschen selbst, weil er merkt, er ist wichtig im Prozess und (er) wird halt auch akzeptiert und die Tätigkeit, die er täglich macht. [...] Je mehr man selbst lösen kann, braucht man zwar weniger Kollegen, aber der Prozess läuft einfach geglätteter ab. (Interview 43, Anwendende)

\subsection{Formale, non-formale und informelle Wege des Kompetenzaufbaus sind bedeutsam}

In Bezug auf die Entwicklung der notwendigen Kompetenzen wurden von den befragten Experten sowohl formale als auch non-formale und informelle Wege der Kompetenzentwicklung benannt. Die Ergebnisse legen nahe, dass bei der Vermittlung der notwendigen Kompetenzen bei Einfachbeschäftigten vor allem betriebsinterne Kurse und das Anlernen, Unterweisen und Informieren durch Kollegen und Vorgesetzte von Bedeutung sind. Beide Kategorien wurden über alle Expertengruppen hinweg häufig genannt, was ein Indiz für die Relevanz dieser Formate sein könnte. Dabei sollte jedoch angemerkt werden, dass diese Wege der Kompetenzvermittlung bereits heute in praktisch orientierten $\mathrm{Be}$ rufen weit verbreitet sind (Kortsch et al. 2019) und sich die Nennung der Kategorien daher auch aus der heute erlebten Realität speisen könnte.

An die Seite der traditionellen Wege der Weiterbildung treten non-formale Wege der Kompetenzentwicklung, die durch digitale Technologien ermöglicht werden, wie das digitale Anlernen, Unterweisen und Infomieren oder das selbstgesteuerte Lernen mit Medien. Neben formalen und 
non-formalen Wegen der Kompetenzentwicklung werden mit dem Lernen im Prozess der Arbeit und dem digitalen Lernen im Prozess der Arbeit auch Wege des informellen Lernens benannt. Beim Lernen im Prozess der Arbeit fällt auf, dass diese Kategorie vor allem von Wissenschaftler/ innen benannt wird. Eine mögliche Ursache hierfür könnte das große Forschungsinteresse zum Thema informelles Lernen und lernförderliche Arbeitsgestaltung sein (Rohs 2020) sowie die Tatsache, dass von Lernenden selbst informelles Lernen häufig nicht als Erweiterung oder Verbesserung des eigenen Wissens oder eigener Fähigkeiten wahrgenommen wird (Kommission der Europäischen Gemeinschaften 2000) und daher ggf. auch im Rahmen der Interviews von Anwendenden nur selten als Weg der Kompetenzentwicklung benannt wurde.

Einschränkend sollte an dieser Stelle berücksichtigt werden, dass die Interviewdaten keinen Aufschluss darüber zulassen, mit welchen Methoden welche Kompetenzen besonders gut aufgebaut werden können. Die am häufigsten benannten Formate wie betriebsinterne Kurse und Anlernen, Unterweisen und Informieren durch Kollegen und Vorgesetzte legen die Vermutung nahe, dass die Interviewteilnehmenden vorranging Formate zum Aufbau der fachlichen Kompetenzen benannt haben.

\subsection{Theoretische Implikationen}

Die vorliegende Studie nimmt erstmalig eine detaillierte Aufgliederung der von Produktionsbeschäftigten benötigten Kompetenzen beim vermehrten Einsatz von DWAS im Arbeitskontext vor. Durch die Identifikation von 18 Kompetenzdimensionen trägt die Studie zu einem besseren Verständnis der Kompetenzanforderungen in der Industrie 4.0 für Produktionsbeschäftigte bei. Durch die Integration unterschiedlicher Expertengruppen wurde ein umfassender Blick auf zukünftig relevante Kompetenzen ermöglicht (vgl. Klug 2011).

Durch die detaillierte Betrachtung der digitalen Kompetenz konnten bekannte Aspekte wie die Bedeutung einer allgemeinen IT-Anwendungskompetenz und Kompetenzen bei Datenschutz und Datensicherheit bestätigt werden. Darüber hinaus konnten weitere relevante Aspekte der digitalen Kompetenz wie die Erstellung digitaler Inhalte, die digitale Problembehebung und die digitale Selbstachtsamkeit identifiziert werden. Damit erweitert diese Studie bestehende Befunde zur Systematisierung digitaler Kompetenzen (z.B. Carretero et al. 2017; Ferrari 2012; Oberländer et al. 2020). Unseres Wissens nach ist dies die erste Studie, die systematisch die Kompetenzanforderungen an Produktionsbeschäftigte im Umgang mit DWAS erfasst. Mit der Betrachtung der Produktionsarbeit geht diese Studie über die häufig betrachteten Untersuchungskontexte der Gesamtgesellschaft (z. B. Carretero et al. 2017; Ferrari 2012) und der
Wissensarbeit (z.B. Oberländer et al. 2020) hinaus. Durch die Ergebnisse unserer Studie hoffen wir, zu einem tieferen Verständnis des Konzepts der digitalen Kompetenz im Arbeitskontext beitragen zu können.

\subsection{Praktische Implikationen}

Die zunehmende Verbreitung digitaler Technologien und die damit einhergehenden Veränderungen von Arbeitsanforderungen machen ein ganzheitliches Kompetenzmanagement in Unternehmen notwendig (Dombrowski und Wagner 2014). Das im Rahmen dieser Studie erstellte Kompetenzmodell kann Unternehmen als Vorlage für die Entwicklung unternehmensspezifischer Kompetenzmodelle für Produktionsbeschäftigte im Umgang mit DWAS dienen und durch unternehmensintern abgeleitete Kompetenzen ergänzt werden.

Dabei ist zu beachten, dass die Bedeutung einzelner Kompetenzen und notwendige Niveaustufen von der Ausgestaltung und Anwendung der Industrie 4.0-Technologien innerhalb des jeweiligen Unternehmens abhängig sind. Werden digitale Technologien als Werkzeuge eingesetzt und bleiben für die Beschäftigten weiterhin große Entscheidungsfreiheiten über den eigenen Arbeitsprozess erhalten, können Kompetenzen wie das Prozessdenken, die Analysefähigkeit oder das Vertreten eines eigenen Standpunktes von größerer Relevanz sein als in einem Szenario, in dem die Arbeitsabläufe ausschließlich von digitalen Technologien vorgegeben werden und Beschäftigten lediglich eine ausführende Tätigkeit zukommt (vgl. Werkzeugszenario und Automatisierungsszenario z.B. Dombrowski et al. 2014; Windelband und Spöttl 2012). Durch die bewusste Gestaltung und die Auswahl der Einsatzszenarien für DWAS (siehe Blumberg und Kauffeld 2020) bieten sich Unternehmen neue Ansätze zur Gestaltung der Einfacharbeit. Dabei können Unternehmen bewusst ein Upgrading (Hirsch-Kreinsen 2016) der Arbeitstätigkeit im Sinne einer Erweiterung der Arbeitsanforderungen fördern und Produktionsbeschäftigten mehr Verantwortung für die Problemlösung übertragen.

In Bezug auf die Kompetenzentwicklung zeigt die Studie, dass Unternehmen sowohl formale als auch non-formale und informelle Wege der Kompetenzentwicklung nutzen können, um die erforderlichen Kompetenzen aufzubauen. Produktionsbeschäftigte haben vorrangig betriebsinterne Kurse und das Anlernen, Unterweisen und Informieren durch Kollegen und Vorgesetzte als Wege der Kompetenzentwicklung benannt. Dies könnte vor allem daran liegen, dass diese Formate bekannt und in der heutigen Produktionsarbeit häufig eingesetzt werden, um Wissen und Fähigkeiten für die Arbeitssituation aufzubauen. Interaktive Lernformate, wie Lernfabriken, die abseits der eigentlichen Arbeitstätigkeit stattfinden, jedoch einen hohen Realitäts- 
bezug haben (Abele et al. 2016, 2017), bieten neben der Vermittlung des notwendigen fachlichen Wissens auch die Chance, Methoden-, Sozial- und Selbstkompetenzen aufzubauen (für einen Überblick über bestehende Publikationen siehe Müller-Frommeyer et al. 2017). So kann beispielsweise der Aufbau von Technikaffinität bzw. der Abbau von Berührungsängsten mit neuen Technologien in Lernfabriken erfolgen (Müller-Frommeyer et al. 2017). Dies wird auch im folgenden Zitat aus den Interviews sichtbar:

Es gibt genügend Programme, heißt Simulationen, die man mit den Mitarbeitern durchspielen könnte, um denen einfach die Ängste zu nehmen. (Interview 45, Anwendende)

Darüber hinaus bietet die Integration von DWAS in den Arbeitsalltag eine Möglichkeit, um stärker als bisher digitale Lernformate auch für Produktionsbeschäftigte zu realisieren. Bei digitalen Lernformaten ändert sich nicht nur das Format des Lernens, sondern teilweise auch die Rolle der Beschäftigten. Lernprozesse werden nicht mehr nur von außen angeordnet oder angestoßen, sondern können nun auch von Beschäftigten selbst initiiert werden (Melzer et al. 2019; Metternich et al. 2018). Um auch in zeitlich eng getakteten Arbeitsrealitäten wie in der industriellen Produktion digitales Lernen zu ermöglichen, können Wissensbausteinen bedarfsgerecht in Form von Lern- bzw. Wissensnuggets bereitgestellt werden (Acatech 2016). Bei Wissensnuggets handelt es sich um kurze, alleinstehende Lerneinheiten, die ein festes Lernziel anstreben und von den Mitarbeitenden bei Bedarf abgerufen und in bewegten Bildern angeschaut und durchgeführt werden können (Bauer et al. 2018; Bailey et al. 2006). Trotz aller (neuen) Möglichkeiten der betrieblichen Weiterbildung sollte an dieser Stelle berücksichtigt werden, dass bei an- und ungelernten Beschäftigten negative Assoziationen zu Lern- und Prüfungssituationen sowie Prüfungsängste bestehen können (Güth et al. 2018). Diese besondere Gegebenheit muss bei der Umsetzung von Lernangeboten beispielsweise durch den Einsatz von Gamification-Elementen sowie den Verzicht auf umfangreiche Wissenstests und Prüfungssituationen berücksichtigt werden. So können (digitale) Übungslandschaften geschaffen werden, in denen geprobt und nebenbei das Gelingen gemessen und honoriert werden kann (Kauffeld 2016).

\subsection{Limitationen}

Bei der Interpretation und Nutzung der Studienergebnisse sind einige Einschränkungen zu beachten. Die erste Limitation ergibt sich hinsichtlich der Generalisierbarkeit der gefundenen Ergebnisse. In die Studie wurden unterschiedliche Expertengruppen einbezogen, um ein möglichst breites Bild zukünftig benötigter Kompetenzen für Beschäftigte in der Produktion beim Einsatz von DWAS zu erhalten. Die
Teilnehmenden aus den Gruppen der betrieblichen Praxis (Planer/innen, Betriebsräte, Anwendende) stammten jedoch alle aus einem Unternehmen. Da die Teilnehmenden unterschiedlichen organisatorischen Einheiten angehörten, kann davon ausgegangen werden, dass diese unterschiedliches Wissen und Erfahrungen bei der Beantwortung der Fragen eingebracht haben (Dougherty 1992; Fleck 1979; Tortoriello et al. 2012). Dies zeigt sich auch in den breiten Antworten, die wir in den einzelnen Expertengruppen erhalten haben. Dennoch könnte unternehmensinternes Wissen zu bekannten bzw. als gewollt erachteten Nutzungs- und Anwendungsmöglichkeiten von DWAS das Antwortverhalten beeinflusst haben (z.B. im Sinne der sozialen Erwünschtheit, siehe unten). Bei der Übertragung des Kompetenzmodells in die betriebliche Praxis sollte daher ein Abgleich mit unternehmenseigenen Strategien zum Einsatz von DWAS erfolgen.

Weitere Limitationen ergeben sich aus der Methode der Datengewinnung durch persönliche Interviews. In einem solchen Befragungssetting kann sozial erwünschtes Antwortverhalten verstärkt werden (Grimm 2010). Dabei wird die eigene Person, aber auch der eigene soziale Kontext (beispielsweise das Unternehmen) so dargestellt, wie es als sozial erwünscht oder akzeptiert empfunden wird (Bergen und Labonté 2020). Wir halten diesen Aspekt jedoch für vernachlässigbar, da es sich um eine zukunftsorientierte Fragestellung handelt, zu der (noch) keine gesamtgesellschaftlichen Bewertungen vorliegen. Dies zeigt sich auch in den Interviews. Die Teilnehmenden äußerten sich differenziert zu den einzelnen Fragestellungen und nahmen unterschiedliche Positionen ein. An die interviewende Person wird eine doppelte Anforderung gestellt. Zum einen soll eine größtmögliche Unvoreingenommenheit bestehen, um das Antwortverhalten der Teilnehmenden nicht durch die eigene Meinung oder bestehende Erkenntnisse zu lenken. Zum anderen ist eine Auseinandersetzung mit der Literatur notwendig, um Forschungsfragen zu entwickeln und eine Sensibilität für die Thematik aufzubauen (Witzel 1985). Um dies zu berücksichtigen, wurden die Forschungsfragen zunächst vorläufig formuliert und lediglich eine grobe Sichtung relevanter Literatur vorgenommen. Erst ab der Datenaufbereitung erfolgte eine detaillierte Auseinandersetzung mit der bestehenden Forschung.

Weitere Limitationen ergeben sich durch die Datenaufbereitung. Bei der Erstellung von Kategorien handelt es sich um einen subjektiven und interpretativen Prozess, der wesentlich von Wissen, Erfahrung und Denkmustern der Forschenden beeinflusst wird (Kuckartz 2016; Mayring 2008). Um dem zu begegnen, wurde besonders auf ein strukturiertes und transparentes Vorgehen geachtet (u. a. durch die Erstellung und iterative Überarbeitung eines Kodierleitfadens mit Kodierregeln und Kodierbeispielen sowie Erläuterungen zur Abgrenzung verwandter Kategorien, Kuckartz 
und Rädiker 2019). Dadurch konnte eine hohe Güte bei der Vergabe der Kategorien sichergestellt werden (siehe Kapitel 3.3).

Bei der Aufbereitung der Daten und der Erstellung der Kategoriensysteme wurde auf bestehende und etablierte Rahmenmodelle zurückgegriffen (Kompetenzfacetten: Fach-, Methoden-, Sozial- und Selbstkompetenz; Wege der Kompetenzentwicklung: formal, non-formal, informell). Kritisch kann an dieser Stelle angemerkt werden, dass es sich bei dem genutzten Kompetenzmodell um ein generisches Modell handelt und Hypothese 1 zur Passung des Modells für die Systematisierung der ermittelten Kompetenzen damit kaum falsifizierbar war. Auch andere generische Kompetenzmodelle (z.B. Kompetenzatlas Heyse 2007) wären für die Systematisierung der Kompetenzanforderungen denkbar gewesen. Das hier genutzte Rahmenmodell mit der schlichten Unterteilung in Fach-, Methoden-, Sozial- und Selbstkompetenz (vgl. Kauffeld 2006; Kauffeld und Paulsen 2018; Sonntag und Schaper 1999) erschien uns für eine einfache Übertragbarkeit der Ergebnisse in die unternehmerische Praxis am geeignetsten.

\subsection{Schlussfolgerungen und zukünftiger Forschungsbedarf}

Die vorliegende Studie leistet mit der Betrachtung der zunehmenden Anwendung von DWAS im Produktionskontext einen Beitrag zur frühzeitigen Identifikation zukünftig relevanter Kompetenzanforderungen. Vor allem durch die detaillierte Betrachtung der erforderlichen digitalen Kompetenzen und die Beschreibung der sieben Dimensionen Informations- und Datenverarbeitungskompetenz, Erstellung digitaler Inhalte, Datenschutz und Systemsicherheit, digitale Problembehebung, allgemeine IT-Anwendungskompetenz, digitale Kommunikation und Kooperation sowie digitale Selbstachtsamkeit wurden Ansatzpunkte für die weitere theoretische Erschließung des Konstruktes digitaler Kompetenzen geschaffen. Für die Praxis bieten diese detaillierten Beschreibungen eine Orientierung und die Möglichkeit, mit Kompetenzentwicklungsmaßnahmen gezielt die unterschiedlichen Kompetenzen aufzubauen. Zukünftige Studien sollten an diesen Befunden anknüpfen und die identifizierten Kompetenzen sowie Veränderungen der Arbeitsaufgaben im Zuge der Einführung von DWAS durch begleitende Forschung überprüfen und erweitern.

In Bezug auf die Wege der Kompetenzentwicklung zeigt diese Studie, dass etablierte Formen des Lernens durch neue, digital ermöglichte Formate ergänzt und erweitert werden. Digitale Formate des non-formalen Lernens (z. B. digitales Anlernen, Unterweisen und Informieren und das selbstgesteuerte Lernen mit Medien) werden am seltensten von der Gruppe der Anwendenden benannt. Dies könnte ein Anzeichen dafür sein, dass DWAS von Anwendenden primär als Arbeitswerkzeug wahrgenommen werden. Andere Expertengruppen verbinden mit der Einführung und Nutzung von DWAS hingegen nicht nur ein Arbeits-, sondern auch ein Lerngerät, das die Arbeits- und Lernrealität der Produktionsbeschäftigten verändern könnte. Digitale Lernformate und die dadurch weiter zunehmende Verschmelzung von Arbeits- und Lernprozessen, die bisher beschreibend für die Wissensarbeit waren (Clasen 2018), könnten zukünftig auch eine höhere Relevanz in Produktionsbereichen erfahren. Dabei ist zu berücksichtigen, dass die Wissensarbeit häufig von einer großen Handlungs- und Entscheidungsfreiheit geprägt ist, die es den Beschäftigten ermöglicht, Zeit für die Recherche, Aneignung und Erprobung des neuen Wissens aufzubringen (Clasen 2018). Wenn auch in der Produktionsarbeit Lern- und Arbeitsprozesse immer stärker miteinander verschmelzen, müssen bei der Gestaltung von Produktionsarbeit die notwendigen Freiheitsgrade geschaffen werden, um die neuen Formen des Lernens zu ermöglichen. Neben der Arbeitsgestaltung spielen auch betriebliche Regelungen über Arbeits- und Lernzeiten eine bedeutsame Rolle, um beispielsweise das selbstgesteuerte Lernen mit Medien außerhalb des Arbeitsortes zu ermöglichen. Produktionsbeschäftigte müssen auf diese veränderten Lernrealitäten vorbereitet werden.

Offen bleiben die Fragen, mit welchen Kompetenzentwicklungsmaßnahmen welche zukünftig relevanten Kompetenzen besonders gut aufgebaut werden können und wie der Transfer vom Lernen in die Arbeit bestmöglich gestaltet werden kann. Diesen Fragen sollte in zukünftigen Studien nachgegangen werden, um Kompetenzentwicklungsmaßnahmen sowohl aus Sicht der Mitarbeitenden als auch des Unternehmens effizient, effektiv und erfolgreich gestalten zu können.

Funding Open Access funding enabled and organized by Projekt DEAL.

Open Access Dieser Artikel wird unter der Creative Commons Namensnennung 4.0 International Lizenz veröffentlicht, welche die Nutzung, Vervielfältigung, Bearbeitung, Verbreitung und Wiedergabe in jeglichem Medium und Format erlaubt, sofern Sie den/die ursprünglichen Autor(en) und die Quelle ordnungsgemäß nennen, einen Link zur Creative Commons Lizenz beifügen und angeben, ob Änderungen vorgenommen wurden.

Die in diesem Artikel enthaltenen Bilder und sonstiges Drittmaterial unterliegen ebenfalls der genannten Creative Commons Lizenz, sofern sich aus der Abbildungslegende nichts anderes ergibt. Sofern das betreffende Material nicht unter der genannten Creative Commons Lizenz steht und die betreffende Handlung nicht nach gesetzlichen Vorschriften erlaubt ist, ist für die oben aufgeführten Weiterverwendungen des Materials die Einwilligung des jeweiligen Rechteinhabers einzuholen.

Weitere Details zur Lizenz entnehmen Sie bitte der Lizenzinformation auf http://creativecommons.org/licenses/by/4.0/deed.de. 


\section{Literatur}

Abele, E., Bauerdick, C. J., Strobel, N., \& Panten, N. (2016). ETA learning factory: a holistic concept for teaching energy efficiency in production. Procedia CIRP, 54, 83-88. https://doi.org/10. 1016/j.procir.2016.06.051.

Abele, E., Chryssolouris, G., Sihn, W., Metternich, J., ElMaraghy, H., Seliger, G., Sivard, G., ElMaraghy, W., Hummel, V., Tisch, M., \& Seifermann, S. (2017). Learning factories for future oriented research and education in manufacturing. CIRP Annals, 66(2), 803-826. https://doi.org/10.1016/j.cirp.2017.05.005.

Acatech (2016). Kompetenzen für Industrie 4.0: Qualifizierungsbedarfe und Lösungsansätze (acatech Position). https://www.acatech. de/wp-content/uploads/2018/03/161202_POS_Kompetenz_Indu strie40_Web.pdf. Zugegriffen: 26.07.2020.

Akremi, L. (2019). Stichprobenziehung in der qualitativen Sozialforschung. In N. Baur \& J. Blasius (Hrsg.), Handbuch Methoden der empirischen Sozialforschung (S. 313-331). Wiesbaden: Springer. https://doi.org/10.1007/978-3-658-21308-4_21.

Baethge-Kinsky, V. (2020). Digitized industrial work: requirements, opportunities, and problems of competence development. Frontiers in Sociology, 5, 447. https://doi.org/10.3389/fsoc.2020. 00033.

Bailey, C., Zalfan, M. T., Davis, H. C., Fill, K., \& Conole, G. (2006). Panning for gold: designing pedagogically-inspired learning nuggets. Journal of Educational Technology \& Society, 9(1), $113-122$.

Bär, D., Bentkämper, P., Berg, A., \& Sager, R. (2020). Digitaltag 2020: Gemeinsam digitale Teilhabe fördern. Digitaltag 2020, Berlin. https://digitaltag.eu/sites/default/files/2020-06/ Pr\%C3\%A4sentation\%20Digitaltag-Auftakt-PK\%2016\%2006 \%202020_Webseite_Studie_final.pdf. Zugegriffen: 30. Aug. 2020.

Bauer, W., Hämmerle, M., Bauernhansl, T., \& Zimmermann, T. (2018). Future Work Lab: Arbeitswelt der Zukunft. In R. Neugebauer (Hrsg.), Fraunhofer-Forschungsfokus. Digitalisierung: Schlüsseltechnologien für Wirtschaft und Gesellschaft (S. 179-195). Berlin, Heidelberg: Springer Vieweg.

Bawden, D. (2008). Origins and concepts of digital literacy. In C. Lankshear \& M. Knobel (Hrsg.), Digital literacies: concepts, policies and practices. New literacies and digital epistemologies, (Bd. 30, S. 17-32). New York: Peter Lang.

Bellmann, L., Dummert, S., Ebbinghaus, M., Krekel, E.M., \& Leber, U. (2015). Qualifizierung von Beschäftigten in einfachen Tätigkeiten und Fachkräftebedarf. Zeitschrift für Weiterbildungsforschung, 38(2), 287-301. https://doi.org/10.1007/s40955-0150022-0.

Bergen, N., \& Labonté, R. (2020). „Everything is perfect, and we have no problems": detecting and limiting social desirability bias in qualitative research. Qualitative Health Research, 30(5), 783-792. https://doi.org/10.1177/1049732319889354.

Blumberg, V. S. L., \& Kauffeld, S. (2020). Anwendungsszenarien und Technologiebewertung von digitalen Werkerassistenzsystemen in der Produktion - Ergebnisse einer Interview-Studie mit Experten aus der Wissenschaft, der Politik und der betrieblichen Praxis. Gruppe. Interaktion. Organisation. Zeitschrift für Angewandte Organisationspsychologie, 51(1), 5-24. https://doi.org/10.1007/ s11612-020-00506-0.

Bornewasser, M., Evers, J., \& Warner, N. (2018). Kompetenzerwerb in vernetzten Strukturen. In M. Bornewasser (Hrsg.), Kompetenzmanagement in Organisationen. Vernetztes Kompetenzmanagement: Gestaltung von Lernprozessen in organisationsübergreifenden Strukturen (S. 11-23). Berlin, Heidelberg, New York: Springer. https://doi.org/10.1007/978-3-662-54954-4_2.

Botthof, A. (2015). Zukunft der Arbeit im Kontext von Autonomik und Industrie 4.0. In A. Botthof \& E. A. Hartmann (Hrsg.), Zukunft der
Arbeit in Industrie 4.0 (S. 3-8). Berlin, Heidelberg, New York: Springer. https://doi.org/10.1007/978-3-662-45915-7_1.

Bouville, G., \& Alis, D. (2014). The effects of lean organizational practices on employees' attitudes and workers' health: evidence from France. The International Journal of Human Resource Management, 25(21), 3016-3037. https://doi.org/10.1080/09585192. 2014.951950.

Brennan, R.L., \& Prediger, D. J. (1981). Coefficient kappa: some uses, misuses, and alternatives. Educational and Psychological Measurement, 41(3), 687-699. https://doi.org/10.1177/ 001316448104100307.

Brockmann, M., Clarke, L., Méhaut, P., \& Winch, C. (2008). Competence-based Vocational Education and Training (VET): the cases of England and France in a European perspective. Vocations and Learning, 1(3), 227-244. https://doi.org/10.1007/s12186-0089013-2.

Brown, R.B. (1993). Meta-competence: a recipe for reframing the competence debate. Personnel Review, 22(6), 25-36.

Carretero, S., Vuorikari, R., \& Punie, Y. (2017). DigComp 2.1: The digital competence framework for citizens with eight proficiency levels and examples of use. EUR, scientific and technical research series, Bd. 28558. Luxembourg: Publications Office.

Cascio, W.F., \& Montealegre, R. (2016). How technology is changing work and organizations. Annual Review of Organizational Psychology and Organizational Behavior, 3(1), 349-375. https://doi. org/10.1146/annurev-orgpsych-041015-062352.

Clasen, E. (2018). Wenn Arbeit und Lernen zusammenfallen: Zum beruflichen Selbstverständnis in der Wissensarbeit. In H. Johns \& G. Vedder (Hrsg.), Organisation von Arbeit und berufsbegleitendem Lernen. Schriftenreihe zur interdisziplinären Arbeitswissenschaft, (Bd. v.10, S. 115-127). Augsburg: Rainer Hampp.

Dehnbostel, P. (2019). Betriebliche Lernorte, Lernräume und Selbstlernarchitekturen in der digitalisierten Arbeitswelt. Magazin erwachsenenbildung.at. Das Fachmedium für Forschung, Praxis und Diskurs. (35/36). http://www.erwachsenenbildung.at/ magazin/19-35u36/meb19-35u36.pdf. Zugegriffen: 2. Aug. 2020.

Dillerup, R., Witzemann, T., Schacht, S., \& Schaller, L. (2019). Planung im digitalen Zeitalter. Controlling \& Management Review, 63(3), 46-53. https://doi.org/10.1007/s12176-019-0011-z.

Dombrowski, U., \& Wagner, T. (2014). Arbeitsbedingungen im Wandel der Industrie 4.0: Mitarbeiterpartizipation als Erfolgsfaktor zur Akzeptanzbildung und Kompetenzentwicklung. ZWF Zeitschrift für wirtschaftlichen Fabrikbetrieb, 109(5), 351-355.

Dombrowski, U., Riechel, C., \& Evers, M. (2014). Industrie 4.0 - Die Rolle des Menschen in der vierten industriellen Revolution. In W. Kersten, H. Koller \& H. Lödding (Hrsg.), Industrie 4.0: Wie intelligente Vernetzung und kognitive Systeme unsere Arbeit verändern. Schriftenreihe der Hochschulgruppe für Arbeits- und Betriebsorganisation e. V. (HAB). (S. 129-153). Berlin: Gito.

Dougherty, D. (1992). Interpretive barriers to successful product innovation in large firms. Organization Science, 3(2), 179-202. https:// doi.org/10.1287/orsc.3.2.179.

Dresing, T., \& Pehl, T. (2018). Praxisbuch Interview, Transkription \& Analyse: Anleitungen und Regelsysteme für qualitativ Forschende. Marburg: Eigenverlag.

Elkmann, N., Berndt, D., Leye, S., Richter, K., \& Mecke, R. (2015). Arbeitssysteme der Zukunft. In M. Schenk (Hrsg.), Produktion und Logistik mit Zukunft (S. 49-149). Berlin, Heidelberg, New York: Springer. https://doi.org/10.1007/978-3-662-48266-7_2.

Erpenbeck, J. (2012). Was sind Kompetenzen. In W.G. Faix (Hrsg.), Kompetenz: Festschrift-Prof. Dr. John Erpenbeck zum 70. Geburstag. Kompetenz, Persönlichkeit, Bildung/School of International Business and Entrepreneurship, (Bd. 4, S. 1-57). Stuttgart: Steinbeis-Ed.

Erpenbeck, J., Grote, S., \& Sauter, W. (2017). Einführung. In J. Erpenbeck, L. v. Rosenstiel, S. Grote \& W. Sauter (Hrsg.), Handbuch Kompetenzmessung: Erkennen, verstehen und bewerten von 
Kompetenzen in der betrieblichen, pädagogischen und psychologischen Praxis (S. IX-XXXVIII). Stuttgart: Schäffer-Poeschel.

Evers, M., Krzywdzinski, M., \& Pfeiffer, S. (2019). Wearable Computing im Betrieb gestalten. Arbeit, 28(1), 3-27. https://doi.org/10. 1515/arbeit-2019-0002.

Ferrari, A. (2012). Digital competence in practice: an analysis of frameworks (JRC technical reports). Luxembourg. https://pdfs. semanticscholar.org/851f/ebe72df176a16ad6e26b00ff5df35520da 34.pdf. Zugegriffen: 27. Juli 2020.

Fischer, A., \& Neubert, J.C. (2015). The multiple faces of complex problems: a model of problem solving competency and its implications for training and assessment. Journal of Dynamic Decision Making, 1(6), 1-13. https://doi.org/10.11588/jddm.2015.1.23945.

Fleck, L. (1979). Genesis and development of a scientific fact. Sociology of science (3. Aufl.). Chicago: University of Chicago Press. Translated by Frederick Bradley and Thaddeus J. Trenn

Gilster, P. (1997). Digital literacy. New York: Wiley Computer Publishing.

Gläser, J., \& Laudel, G. (2010). Experteninterviews und qualitative Inhaltsanalyse als Instrumente rekonstruierender Untersuchungen (4. Aufl.). Wiesbaden: VS. Lehrbuch

Grimm, P. (2010). Social desirability bias. In J. N. Sheth \& N. K. Malhotra (Hrsg.), Wiley international encyclopedia of marketing. Chichester: John Wiley \& Sons Ltd. https://doi.org/10.1002/ 9781444316568.wiem02057.

Güth, S., Decius, J., Horvat, D., Schaper, N., \& Virgillito, A. (2018). Strategisches Kompetenzmanagement von Produktionsbeschäftigen: Innovations- und Wachstumsimpulse in nichtforschungsintensiven kleinen und mittleren Unternehmen. In D. Ahrens \& G. Molzberger (Hrsg.), Kompetenzmanagement in Organisationen. Kompetenzentwicklung in analogen und digitalisierten Arbeitswelten: Gestaltung sozialer, organisationaler und technologischer Innovationen (S. 31-49). Berlin, Heidelberg, New York: Springer. https://doi.org/10.1007/978-3-662-54956-8_4.

Hammermann, A., \& Stettes, O. (2016). Qualifikationsbedarf und Qualifizierung: Anforderungen im Zeichen der Digitalisierung. IW policy paper, Institut der deutschen Wirtschaft 3/2016. Köln.

Hesse, F., Care, E., Buder, J., Sassenberg, K., \& Griffin, P. (2015). A framework for teachable collaborative problem solving skills. In P. Griffin \& E. Care (Hrsg.), Assessment and teaching of 21st century skills (S. 37-56). Dordrecht: Springer. https://doi.org/10. 1007/978-94-017-9395-7_2.

Heyse, V. (2007). Strategien - Kompetenzanforderungen - Potenzialanalysen. In V. Heyse \& J. Erpenbeck (Hrsg.), Kompetenzmanagement: Methoden, Vorgehen, $K O D E^{\circledR}$ und $K O D E^{\circledR} X$ im Praxistest (S. 11-180). Münster, New York, München, Berlin: Waxmann.

Hillebrand, A. (2018). Welche Kompetenzen zeichnen einen Experten aus? Entwicklung eines expertenspezifischen Kompetenzmodells in einem produzierenden Unternehmen. AutoUni-Schriftenreihe, Bd. 126. Wiesbaden: Springer. Dissertation

Hinrichsen, S., Riediger, D., \& Unrau, A. (2016). Assistance systems in manual assembly. In F.-J. Villmer \& E. Padoano (Hrsg.), 01. Production engineering and management: Proceedings 6th international conference, September 29 and 30, 2016 Lemgo, Germany. Publication series in direct digital manufacturing, (Bd. 2016, S. 3-14). Lemgo, Triest: Hochschule Ostwestfalen-Lippe; Università.

Hirsch-Kreinsen, H. (2016). Digitization of industrial work: development paths and prospects. J Labour Market Res, 49(1), 1-14. https://doi.org/10.1007/s12651-016-0200-6.

Hirsch-Kreinsen, H. (2017). Digitalisierung industrieller Einfacharbeit. Arbeit, 26(1), 9. https://doi.org/10.1515/arbeit-2017-0002.

International Organization for Standardization (ISO) (2015). Quality management systems-requirements. (9001:2015)

Kauffeld, S. (2006). Kompetenzen messen, bewerten, entwickeln: Ein prozessanalytischer Ansatz, für Gruppen. Betriebswirtschaftli- che Abhandlungen: Neue Folge, Bd. 128. Stuttgart: SchäfferPoeschel.

Kauffeld, S. (2016). Nachhaltige Personalentwicklung und Weiterbildung: Betriebliche Seminare und Trainings entwickeln, Erfolge messen, Transfer sichern. Bd. 2. Berlin, Heidelberg, New York: Springer.

Kauffeld, S., \& Grote, S. (2019). Personalentwicklung. In S. Kauffeld (Hrsg.), Arbeits-, Organisations- und Personalpsychologie für Bachelor (S. 167-210). Berlin, Heidelberg, New York: Springer. https://doi.org/10.1007/978-3-662-56013-6_7.

Kauffeld, S., \& Maier, G. W. (2020). Digitalisierte Arbeitswelt. Gruppe. Interaktion. Organisation. Zeitschrift für Angewandte Organisationspsychologie (GIO), 51(1), 1-4. https://doi.org/10.1007/ s11612-020-00508-y.

Kauffeld, S., \& Paulsen, H.F.K. (2018). Kompetenzmanagement in Unternehmen: Kompetenzen beschreiben, messen, entwicklen und nutzen. Arbeits-, Organisations- und Wirtschaftspsychologie (1. Aufl.). Stuttgart: Kohlhammer.

Kauffeld, S., Handke, L., \& Straube, J. (2016). Verteilt und doch verbunden: Virtuelle Teamarbeit. Gruppe. Interaktion. Organisation. Zeitschrift für Angewandte Organisationspsychologie (GIO), 47(1), 43-51. https://doi.org/10.1007/s11612-016-0308-8.

Klug, A. (2011). Analyse des Personalentwicklungsbedarfs. In J. Ryschka (Hrsg.), Praxishandbuch Personalentwicklung (S. 35-91). Wiesbaden: Springer.

Kommission der Europäischen Gemeinschaften (2000). Memorandum über Lebenslanges Lernen. Brüssel. https://www.die-bonn. de/esprid/dokumente/doc-2000/EU00_01.pdf. Zugegriffen: 27. Sept. 2020.

Kortsch, T., Schulte, E.-M., \& Kauffeld, S. (2019). Learning @ work: informal learning strategies of German craft workers. European Journal of Training and Development, 43(5/6), 418-434. https:// doi.org/10.1108/EJTD-06-2018-0052.

Kuckartz, U. (2016). Qualitative Inhaltsanalyse: Methoden, Praxis, Computerunterstützung. Grundlagentexte Methoden (3. Aufl.). Weinheim, Basel: Beltz Juventa.

Kuckartz, U., \& Rädiker, S. (2019). Analyzing qualitative data with $M A X Q D A:$ text, audio, and video

Lasi, H., Fettke, P., Kemper, H.-G., Feld, T., \& Hoffmann, M. (2014). Industrie 4.0. Wirtschaftsinformatik, 56(4), 261-264. https://doi. org/10.1007/s11576-014-0424-4.

Le Deist, F. D., \& Winterton, J. (2005). What is competence? Human Resource Development International, 8(1), 27-46. https://doi.org/ $10.1080 / 1367886042000338227$.

Leimeister, J. M., \& David, K. (2019a). Lernen im digitalen Zeitalter - Einblicke in aktuelle Erkenntnisse aus der Forschung und Praxis. In J. M. Leimeister \& K. David (Hrsg.), Kompetenzmanagement in Organisationen. Chancen und Herausforderungen des digitalen Lernens: Methoden und Werkzeuge für innovative LehrLern-Konzepte (1. Aufl. S. 3-8). https://doi.org/10.1007/978-3662-59390-5 1.

Leimeister, J. M., \& David, K. (2019b). Trends und Entwicklungen des Lernens im digitalen Zeitalter. In J. M. Leimeister \& K. David (Hrsg.), Kompetenzmanagement in Organisationen. Chancen und Herausforderungen des digitalen Lernens: Methoden und Werkzeuge für innovative Lehr-Lern-Konzepte (1. Aufl. S. 223-230). https://doi.org/10.1007/978-3-662-59390-5_12.

Levy, F. (2010). How technology changes demands for human skills papers. OECD education working papers, Bd. 45.

Mayring, P. (2008). Neuere Entwicklungen in der qualitativen Forschung und der Qualitativen Inhaltsanalyse. In P. Mayring \& M. Gläser-Zikuda (Hrsg.), Praxis der Qualitativen Inhaltsanalyse (S. 7-19). Weinheim: Beltz.

Melzer, A., Heim, Y., Sanders, T., \& Bullinger-Hoffmann, A.C. (2019). Zukunft des Kompetenzmanagements. In A.C. BullingerHoffmann (Hrsg.), Zukunftstechnologien und Kompetenzbedarfe 
(S. 11-24). Berlin, Heidelberg, New York: Springer. https:// doi.org/10.1007/978-3-662-54952-0_2.

Metternich, J., Hertle, C., Tisch, M., Jokovic, B., König, C., Bruder, R., Weber, C., Teneberg, R., \& Ardelt, T. (2018). Betriebliche Kompetenzentwicklungsansätze zur Weiterentwicklung fachlichmethodischer und sozial-kommunikativer Kompetenzen in produktionsnahen Bereichen. In D. Ahrens \& G. Molzberger (Hrsg.), Kompetenzmanagement in Organisationen. Kompetenzentwicklung in analogen und digitalisierten Arbeitswelten: Gestaltung sozialer, organisationaler und technologischer Innovationen (S. 73-87). Berlin, Heidelberg, New York: Springer. https:// doi.org/10.1007/978-3-662-54956-8_6.

Misbah, Z., Gulikers, J., \& Mulder, M. (2019). Competence and knowledge development in competence-based vocational education in Indonesia. Learning Environments Research, 22(2), 253-274. https://doi.org/10.1007/s10984-018-9276-y.

mmb Institut (2019). Auf dem Weg zum Assisted Learning? Digitale Lernanwendungen werden informeller und intelligenter: Ergebnisse der 13. Trendstudie „mmb Learning Delphi“. https://www. mmb-institut.de/wp-content/uploads/mmb-Trendmonitor_20182019.pdf. Zugegriffen: 26. Aug. 2020.

Moore, D. R., Cheng, M., \& Dainty, A. R. (2002). Competence, competency and competencies: performance assessment in organisations. Work Study, 51(6), 314-319. https://doi.org/10.1108/ 00438020210441876.

Mulder, M. (2017). Workplace learning and competence development. The Journal of Agricultural Education and Extension, 23(4), 283-286. https://doi.org/10.1080/1389224X.2017.1348030.

Müller-Frommeyer, L. C., Aymans, S. C., Bargmann, C., Kauffeld, S., \& Herrmann, C. (2017). Introducing competency models as a tool for holistic competency development in learning factories: challenges, example and future application. Procedia Manufacturing, 9, 307-314. https://doi.org/10.1016/j.promfg.2017.04.015.

Niehaus, J. (2017). Mobile Assistenzsysteme für Industrie 4.0: Gestaltungsoptionen zwischen Autonomie und Kontrolle. https://d-nb. info/1175041157/34. Zugegriffen: 26. Juli 2020.

Oberländer, M., Beinicke, A., \& Bipp, T. (2020). Digital competencies: a review of the literature and applications in the workplace. Computers \& Education, 146, 103752. https://doi.org/10.1016/j. compedu.2019.103752.

OECD (2007). Terms, concepts and models for analysing the value of recognition programmes. Wien. http://www.oecd.org/education/ skills-beyond-school/41834711.pdf. Zugegriffen: 31.07.2020.

Okoli, C., \& Pawlowski, S. D. (2004). The Delphi method as a research tool: an example, design considerations and applications. Information \& Management, 42(1), 15-29. https://doi.org/10.1016/j. im.2003.11.002.

Ostermeier, M., Hesse, C., Mühlbradt, T., \& Kuhlang, P. (2018). Anforderungen an Digitalkompetenz in der industriellen Produktion. ZWF Zeitschrift für wirtschaftlichen Fabrikbetrieb, 113(10), 633-636. https://doi.org/10.3139/104.111981.

Pfeiffer, S., Lee, H., Zirnig, C., \& Suphan, A. (2016). Industrie 4.0 - Qualifizierung 2025. Frankfurt am Main. https://www. sabine-pfeiffer.de/files/downloads/2016-Pfeiffer-Industrie40Qualifizierung2025.pdf. Zugegriffen: 2. Apr. 2021.

Promotorengruppe Kommunikation der Forschungsunion Wirtschaft - Wissenschaft (2013). Umsetzungsempfehlungen für das $\mathrm{Zu}$ kunftsprojekt Industrie 4.0. Abschlussbericht des Arbeitskreises Industrie 4.0. Berlin. https://www.bmbf.de/files/Umsetzungsemp fehlungen_Industrie4_0.pdf. Zugegriffen: 26. Juli 2020.

Qu, S. Q., \& Dumay, J. (2011). The qualitative research interview. Qualitative Research in Accounting \& Management, 8(3), 238-264. https://doi.org/10.1108/11766091111162070.

Rädiker, S., \& Kuckartz, U. (2019). Analyse qualitativer Daten mit MAXQDA: Text, Audio und Video. Wiesbaden: Springer.
Renner, K.-H., \& Jacob, N.-C. (2020). Das Interview: Grundlagen und Anwendung in Psychologie und Sozialwissenschaften. Basiswissen Psychologie (1. Aufl.).

Rohs, M. (2020). Informelles Lernen und berufliche Bildung. In R. Arnold, A. Lipsmeier \& M. Rohs (Hrsg.), Handbuch Berufsbildung (S. 441-454). Wiesbaden: Springer.

Saracevic, T. (2006). Relevance: a review of the literature and a framework for thinking on the notion in information science: part II: nature and manifestations of relevance. Journal of the American Society for Information Science, 30, 1915-1933. https://doi.org/ 10.1016/S0065-2830(06)30001-3.

Schaper, N. (2004). Fach-, Methoden- und Sozialkompetenz durch arbeitsbezogenes Lernen in der betrieblichen Ausbildung. In B.S. Wiese (Hrsg.), Individuelle Steuerung beruflicher Entwicklung: Kernkompetenzen in der modernen Arbeitswelt (S. 197-222). Frankfurt a.M.: Campus.

Schoppek, W., \& Fischer, A. (2015). Complex problem solving-single ability or complex phenomenon? Frontiers in Psychology. https:// doi.org/10.3389/fpsyg.2015.01669.

Schöpper-Grabe, S., \& Vahlhaus, I. (2019). Grundbildung und Weiterbildung für Geringqualifizierte (IW-Trends - Vierteljahresschrift zur empirischen No. 1). Köln. https://www.iwkoeln.de/fileadmin/ user_upload/Studien/IW-Trends/PDF/2019/IW-Trends_201901-03_Grundbildung_Geringqualifizierte_.pdf. Zugegriffen: 24.08.2020.

Shippmann, J.S., Ash, R. A., Batjtsta, M., Carr, L., Eyde, L.D., Hesketh, B., Kehoe, J., Pearlman, K., Prien, E.P., \& Sanchez, J.I. (2000). The practice of competency modeling. Personnel Psychology, 53(3), 703-740. https://doi.org/10.1111/j.1744-6570.2000. tb00220.x.

Software, V.E.R. B.I. (2017). MAXQDA 2018 [Computer software]. Berlin. maxqda.com.

Sonntag, K., \& Schaper, N. (1999). Personale Verhaltens- und Leistungsbedingungen. In C. Hoyos \& D. Frey (Hrsg.), Arbeits- und Organisationspsychologie: Ein Lehrbuch. Angewandte Psychologie, (Bd. 1, S. 298-312). Weinheim: Beltz.

Steigleder, S. (2008). Die strukturierende qualitative Inhaltsanalyse im Praxistest: eine konstruktiv kritische Studie zur Auswertungsmethodik von Philipp Mayring. Marburg: Tectum.

Tortoriello, M., Reagans, R., \& McEvily, B. (2012). Bridging the knowledge gap: the influence of strong ties, network cohesion, and network range on the transfer of knowledge between organizational units. Organization Science, 23(4), 1024-1039. https:// doi.org/10.1287/orsc. 1110.0688 .

Ulber, M., \& Remmers, F. (2019). Die Analyse der Arbeitsfähigkeit in der digitalisierten Produktion - Anforderungen an ein neues Instrumentarium. In J. von Garrel (Hrsg.), Weiterbildung und Forschung der SRH Fernhochschule - The Mobile University. Digitalisierung der Produktionsarbeit: Arbeitsfähig sein und bleiben (1. Aufl. S. 113-137). https://doi.org/10.1007/978-3-658-27703$1 \_8$.

Wicht, A., Lechner, C., \& Rammstedt, B. (2018). Wie steht es um die Digitalkompetenz Deutscher Erwachsener? Eine empirische Analyse mit dem Programme for the international Assessment of Adult Competencies (PIAAC). In Berufsverband Deutscher Psychologinnen und Psychologen e.V. (BDP) (Hrsg.), Mensch und Gesellschaft im digitalen Wandel (Psychologie - Gesellschaft Politik) (S. 15-25).

Windelband, L., \& Dworschak, B. (2015). Veränderungen in der industriellen Produktion - Notwendige Kompetenzen auf dem Weg vom Internet der Dinge zu Industrie 4.0. Berufsbildung in Wissenschaft und Praxis (BWP), 44, 26-29.

Windelband, L., \& Spöttl, G. (2012). Diffusion von Technologien in die Facharbeit und deren Konsequenzen für die Qualifizierung am Beispiel des „Internet der Dinge“. In U. Faßhauer, B. Fürstenau \& E. Wuttke (Hrsg.), Schriftenreihe der Sektion Berufs- und Wirtschaftspädagogik der Deutschen Gesellschaft für Erziehungswis- 
senschaft (DGfE). Berufs- und wirtschaftspädagogische Analysen: Aktuelle Forschungen zur beruflichen Bildung (S. 205-219). Opladen: Barbara Budrich.

Witzel, A. (1985). Das problemzentrierte Interview. In G. Jüttemann (Hrsg.), Qualitative Forschung in der Psychologie: Grundfragen, Verfahrensweisen, Anwendungsfelder (S. 227-255). Weinheim: Beltz.

Zeller, B., Achtenhagen, C., \& Föst, S. (2010). Das „Internet der Dinge" in der industriellen Produktion: Studie zu künftigen Qualifikationserfordernissen auf Fachkräfteebene. Nürnberg. https://www. f-bb.de/fileadmin/user_upload/Abschlussbericht_IdD_in_der_ industriellen_Produktion_final.pdf. Zugegriffen: 9. Apr. 2021.

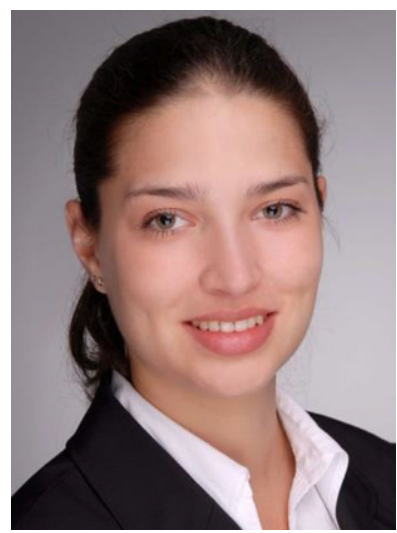

Verena Simone Lore Blumberg promoviert seit 2015 in Kooperation mit einem Mobilitätsunternehmen und wird von Frau Prof. Kauffeld am Lehrstuhl für Arbeits-, Organisations- und Sozialpsychologie der Technischen Universität Braunschweig betreut. Ihr Forschungsschwerpunkt ist die Veränderung von Arbeit durch die Einführung neuer Technologien.

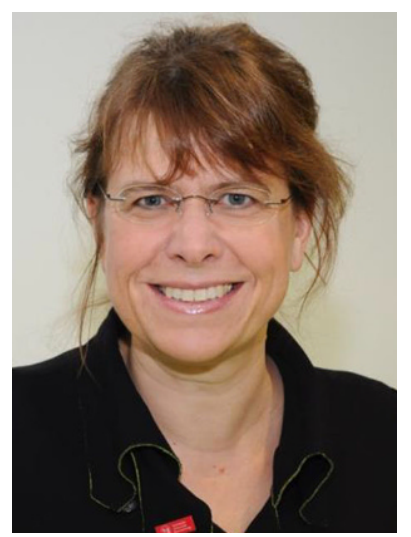

Prof. Dr. Simone Kauffeld ist Inhaberin des Lehrstuhls für Arbeit-, Organisations- und Sozialpsychologie der Technischen Universität Braunschweig. In ihrer Forschungstätigkeit setzt sie sich mit den Themen Kompetenz, Team, Beratung und Führung auseinander. Um ihre Konzepte der Praxis zugänglich zu machen, hat sie 2008 unter Beteiligung der TU Braunschweig die 4ASIDE GmbH gegründet. 